\title{
The first combined checklist of earthworms of the Northeastern Mediterranean region (Clitellata: Megadrili)
}

\author{
T. SZEDERJESI \\ Tímea Szederjesi, Hungarian Natural History Museum, Department of Zoology, H-1088 Budapest, \\ Baross u. 13. E-mail: t.szederjesi@gmail.com
}

\begin{abstract}
The first combined checklist of earthworms of the Balkan Peninsula, Anatolia, the Levant and Cyprus is presented. Out of the 226 taxa, 216 belong to the Holarctic family Lumbricidae. Ten further species are from the families Criodrilidae, Acanthodrilidae, Ocnerodrilidae and Megascolecidae. The widely introduced peregrine species take part with $11.9 \%(27$ taxa). The number of endemics within the studied areas is highest on the Balkan Peninsula (90 taxa). As a result of this study, four synonyms were found, and the subgenus Trapezonscolex Qiu \& Bouché, 1998 was raised to genus level for the former Turkish Eophila species Eo. cavazzutii cavazzutii Omodeo, 1988 and Eo. cavazzutii pascuorum Omodeo, 1988.
\end{abstract}

Keywords. Northeastern Mediterranean, Oligochaeta, species list, Lumbricidae, Megascolecidae, Acanthodrilidae.

\section{INTRODUCTION}

$\mathrm{T}$ he Balkan Peninsula is a relatively welldefined area of Europe. The Black, the Aegean and the Ionian Sea bound it from the eastern, southern and western directions respectively. However, drawing its northern border is quite difficult, the different authors share different concepts (Griffiths et al. 2004, Mahunka et al. 2013). One possible way as proposed here, is to take the valley of the Siret River, the southern border of the Southern Carpathians, the Danube, the Sava, the northern projection of the Dinaric Alps, and the Ćicarija and Učka Mts.

The zoological peculiarities of the Balkans provoked attention as early as the beginning of the 20th century and resulted in intensive researches on the area regarding earthworms as well (Cognetti 1906, Černosvitov 1930). After this longlasting work covering a whole century, one could conceive that the earthworm fauna of the Balkan Peninsula is well-known, but it is important to emphasize that our knowledge on the Balkan countries are quite different, as the monograph of Mršić (1991) reflects it well.
Without any doubt, the former Yugoslav countries are the most well-known areas of the Balkan regarding its eartworm fauna (e.g. Karaman 1972a, Šapkarev 1972a, Karaman \& Stojanović 1996, Stojanović \& Milutinović 2013, Szederjesi 2013a, Trakić et al. 2016, Stojanović et al. 2017a).

Greece (Zicsi \& Michalis 1981, Szederjesi \& Csuzdi 2012a, Szederjesi et al. 2017a) and Bulgaria (Černosvitov 1934a, Zicsi \& Csuzdi 1986, Valchovski 2012, Szederjesi 2013b) are also intensively studied, but there are still huge unexplored areas in the above mentioned countries.

The Albanian earthworm researches are most recent (Dhora 2010, Szederjesi \& Csuzdi 2012b, 2015).

According to the Balkan definition proposed here, the most southern part of Romania also belongs to the Balkan Peninsula, including the Romanian part of Dobruja and the area lying from the southern part of the Carpathians to the Danube. We have only sporadic data from this region (Pop 1949), however two species - Cernosvitovia dobrogeana (Pop, 1938) and C. munteniana Zicsi \& Pop, 1991 - were described from 
here. The European part of Turkey also belongs to the Balkan, the earthworm fauna of this area is lesser known (Valchovski \& Misırlığlu 2017).

Anatolia or Asia Minor forms a large part of Turkey. The Black and the Aegean Sea bound it from the northern and western directions. The Mediterranean Sea, the Taurus Mts and Upper Mesopotamia borders it from south, while the Greater and Lesser Caucasus bound it from northeast and east. The Bosphorus, the Marmara Sea and the Dardanelles separate it from the Balkan Peninsula.

The first organized earthworm collecting trip to Turkey took place at the end of the 1980's and resulted in describing altogether 15 species new to science (Omodeo \& Rota 1989, 1991). Csuzdi et al. (2006) summarized the knowledge on the Turkish earthworm fauna. Since then, the investigations are more or less continuous (Misırlığlu 2008, 2010, 2012, Misırlığlu et al. 2017, Szederjesi et al. 2014a, Szederjesi \& Misırlığlu 2017).

Pavlíček et al. (2007) defines the Levant as "circa $150 \mathrm{~km}$ wide area between the Mediterranean Sea and the Syrian and Arabian deserts. The Taurus Mts borders it from north and the Isthmus of Suez from south".

The earthworm researches in the Levant began at the end of the $19^{\text {th }}$ century (Rosa 1893a, Michaelsen 1901) and continued in the $20^{\text {th }}$ century (Černosvitov 1938a, Omodeo 1956, Zicsi 1985a). The recent studies mainly focused on Israel (Csuzdi \& Pavlíček 1999, 2002, Szederjesi et al. 2013a) and Jordan (Csuzdi \& Pavlíček 2005b, Pavlíček \& Csuzdi 2006a, Szederjesi et al. 2013b).

We have only scarce information on the earthworms of the Aegean islands, most data are sporadic (Cognetti 1906, 1913, Michaelsen 1928, Černosvitov 1934b). The first overview on the Cretan earthworm fauna is from Szederjesi (2017).

Cyprus is the third largest island of the Mediterranean Sea. Its wildlife is species rich, nonetheless we didn't have any information on its earthworm fauna until the 1990's (Michalis 1993) and 2000's (Pavlíček \& Csuzdi 2006b, 2008). Szederjesi et al. (2016) described the first endemic earthworm species from the island. According to Pavlíček \& Csuzdi (2017), the origin of the earthworm fauna of Cyprus probably goes back to the Messinian Salinity Crisis Period, when three, now submerged land bridges - the Misis, Hecataneus and Latakia - connected the island to the Bay of Iskenderun and Syria, due to the lowering of the Mediterranean sea level.

The aim of this paper is to summarise our recent knowledge and present the first checklist of the earthworms of the Balkan Peninsula, Anatolia, Levant and the Aegean Islands aka the Northeastern Mediterranean region.

\section{MATERIAL AND METHODS}

The data were collected by screening the avaible literature. The valid species names are given according to the online database of Csuzdi (2012). In the synonymy list, the original combination of names together with references to the present usage are presented for all species. The comprehensive works of Mršić (1991), Pavlíček et al. (2003), Pavlíček \& Csuzdi (2006b), Valchovski (2012), Csuzdi et al. (2006) and Szederjesi et al. (2017a) are regarded as a basis and only the references published after these works are given.

\section{LIST OF SPECIES}

\section{Family Lumbricidae Rafinesque-Schmaltz, 1815}

\section{Genus Allolobophora Eisen, 1874}

\section{Allolobophora altimontana Mršić, 1982}

\author{
Allolobophora altimontana Mršić, 1982: 58. \\ Hackenberger Kutuzović \& Hackenberger \\ Kutuzović 2013: 3 . \\ Karpatodinariona altimontana: Mršić 1991: 245. \\ Šapkarev 1997: 104.
}

Distribution. Slovenia, Croatia (Hackenberger Kutuzović \& Hackenberger Kutuzović 2013). 


\section{Allolobophora brunnecephala Kvavadze, 1985}

Allolobophora brunnecephala Kvavadze, 1985: 201. Csuzdi et al. 2006: 2. Misirlioğlu et al. 2008: 79. Misirlığlu 2009: 22.

?Allolobophora smaragdina: Omodeo \& Rota 1989: 182. (Csuzdi et al. 2006)

?Allolobophora bellicosa: Omodeo \& Rota 1991: 177. (Csuzdi et al. 2006)

Distribution. Turkey, Georgia (Kvavadze 1985, Csuzdi et al. 2006)

\section{Allolobophora bulgarica Černosvitov, 1934}

Allolobophora bulgarica Černosvitov, 1934a: 74. Trakić et al. 2016: 254.

Cernosvitovia (Cernosvitovia) bulgarica: Mršić 1991: 144.

Cernosvitovia bulgarica: Valchovski 2012: 90. Stojanović et al. 2012: 10.; 2013: 639.

Distribution. Bulgaria (Valchovski 2012).

Remarks. Zicsi (1981a) and Mršić (1991) provisionally placed this species to Cernosvitovia however, Zicsi \& Pop (1991: 126) examining the type specimen revealed that its male pore is located on segment 15 therefore bulgarica belongs to the genus Allolobophora.

\section{Allolobophora carneluttii Mršić, 1990}

Allolobophora (Serbiona) carneluttii Mršić, 1990: 58. Trakić et al. 2016: 254.

Serbiona carneluttii: Mršić 1991: 201. Šapkarev 1997: 104.

Distribution. Serbia (Stojanović et al. 2008).

\section{Allolobophora chlorotica chlorotica (Savigny, 1826)}

Enterion chloroticum Savigny, 1826: 182.

Allolobophora chlorotica: Šapkarev 2001: 100. Pavlíček et al. 2003: 456. Csuzdi et al. 2006: 2. Misırlığlu 2009: 22. Dhora 2010: 82. Stojanović et al. 2012: 9.; 2013: 639.

Allolobophora chlorotica chlorotica: Mršić 1991: 211. Csuzdi \& Pavlíček 2005b: 88. Valchovski 2012: 87.; 2014: 2. Hackenberger Kutuzović \& Hackenberger Kutuzović 2013: 3. Stojanović \&
Milutinović 2013: 148.; 2014: 308. Stojanović et al. 2017a: 180. Szederjesi et al. 2017a: 58. Csuzdi \& Zicsi 2003: 50 (for complete syonymy).

Distribution. widely distributed peregrine species (Csuzdi \& Zicsi 2003).

\section{Allolobophora cryptocystis (Černosvitov, 1935)}

Eophila cryptocystis Černosvitov, 1935a: 265.

Microeophila cryptocystis: Mršić 1991: 223. Šapkarev 1997: 104.

Allolobophora cryptocystis: Csuzdi 2012.

Distribution. Bosnia-Herzegovina (Mršić 1991).

\section{Allolobophora demirkapiae Karaman, 1969}

Allolobophora demirkapiae Karaman, 1969: 80. Szederjesi \& Csuzdi 2012b: 260. Trakić et al. 2016: 254.

Italobalkaniona demirkapiae: Mršić 1991: 163. Šapkarev 1997: 103.

Distribution. Macedonia, Albania (Szederjesi \& Csuzdi 2012b).

\section{Allolobophora dofleini (Ude, 1922)}

Helodrilus (Allolobophora) dofleini Ude, 1922: 157. Allolobophora dofleini: Trakić et al. 2016: 254. Szederjesi et al. 2017a: 58. Stojanović et al. 2017a: 180.; 2017b: 138.

Serbiona dofleini: Mršić, 1991: 180. Šapkarev 1997: 104.; 2001: 100.

Distribution. Serbia, Macedonia, Greece (Szederjesi et al. 2017a).

\section{Allolobophora immaculata Omodeo \& Rota, 1989}

Allolobophora immaculata Omodeo \& Rota, 1989: 181. Csuzdi et al. 2006: 2. Misirlığlu et al. 2008: 79. Misırlığlu 2009: 22.

Distribution. Turkey (Csuzdi et al. 2006).

Allolobophora joncesapkarevi (Blakemore, 2004)

Allolobophora udei Šapkarev, 1972: 120.

Serbiona udei: Mršić 1991: 184. 
Serbiona joncesapkarevi Blakemore, 2004: 78. (nom. nov.)

Allolobophora joncesapkarevi: Csuzdi 2012. Trakić et al. 2016: 255.

Distribution. Macedonia (Mršić 1991).

\section{Allolobophora josapi Blakemore, 2006}

Allolobophora dofleini udei Šapkarev, 1991: 52.; 1997: 104.

Allolobophora josapi Blakemore, 2006: 5. (nom. nov.), Trakić et al. 2016: 255.

Distribution. Macedonia (Šapkarev 1991).

\section{Allolobophora kosowensis Karaman, 1968}

Allolobophora kosowensis Karaman, 1968: 50.

Serbiona kosowensis kosowensis: Mršić 1991: 191. Šapkarev 1997: 104.

Eophila kosowensis: Šapkarev 2002: 304.

Allolobophora kosowensis kosowensis: Stojanović \& Milutinović 2014: 308. Milutinović et al. 2015: 473. Trakić et al. 2016: 255. Stojanović et al. 2017a: 180. Szederjesi et al. 2017a: 58.

Allolobophora kosowensis montenegrina Šapkarev, 1975a: 33. Stojanović \& Milutinović 2013: 148. Trakić et al. 2016: 255. syn. nov.

Serbiona kosowensis montenegrina: Mršić 1991: 192. Šapkarev 1997: 104.

Distribution. Serbia, Montenegro, Greece (Szederjesi et al. 2017a).

Remarks. A. kosowensis kosowensis was described withouth having tubercles but, according to the original description the worms were not fully adult ("Der Clitellus ist schwach entwickelt" = slightly developed). Later, Šapkarev (1975a) described the subspecies kosowensis montenegrina with the same clitellar position as for the typical subspecies but tubercles on 44-50, 51. Until kosowensis kosowensis possessed spermathecae, they were lacking in $k$. montenegrina. Mršić (1991) questioned the lack of tubercles in $A$. kosowensis kosowensis and wrote that kosowensis montenegrina has spermathecae in 10,11 (same as in the typical form). Therefore morphologically the two subspecies are completely identical but represent different developmental stages.

\section{Allolobophora leoni Michaelsen, 1891}

Allolobophora leoni Michaelsen, 1891: 15. Csuzdi et al. 2006: 4. Misırlığlu et al. 2008: 79. Misırlığlu 2009: 22. Valchovski 2012: 87. Stojanović et al. 2012: 9.; 2013: 639.; 2017a: 180. Stojanović \& Milutinović 2014: 308.

Pannoniona leoni: Mršić 1991: 227.

Distribution. Central-Europe and the eastern shore of the Black Sea (Csuzdi \& Zicsi 2003).

\section{Allolobophora macedonica Šapkarev, 1977}

Eophila macedonica Šapkarev, 1977a: 91.

Italobalkaniona macedonica: Mršić 1991: 166. Šapkarev 1997: 103.

Allolobophora macedonica: Csuzdi 2012. Trakić et al. 2016: 256.

Distribution. Macedonia (Mršić 1991).

\section{Allolobophora matjasici Mršić, 1990}

Allolobophora (Serbiona) matjasici Mršić, 1990: 57.

Serbiona matjasici: Mršić 1991: 199. Šapkarev 1997: 104.

Allolobophora matjasici: Trakić et al. 2016: 256.

Distribution. Serbia (Stojanović et al. 2008).

\section{Allolobophora mayeri Mršić, 1990}

Allolobophora (Serbiona) mayeri Mršić, 1990: 56.

Serbiona mayeri: Mršić 1991: 203. Šapkarev 1997: 104.

Allolobophora mayeri: Trakić et al. 2016: 256. 1991).

Distribution. Bosnia-Herzegovina (Mršić

Allolobophora mehadiensis mehadiensis Rosa, 1895

Allolobophora mehadiensis Rosa, 1895: 3. Stojanović et al. 2012: 9.

Serbiona mehadiensis mehadiensis: Mršić 1991: 185. Šapkarev 1997: 104. Valchovski 2012: 98.

Serbiona pannonica (Cognetti, 1906): Mršić 1991: 198. Šapkarev 1997: 104. (Csuzdi 2012)

Allolobophora mehadiensis mehadiensis: Stojanović \& Milutinović 2014: 308. 
Distribution. Hungary, Romania, Serbia, Bulgaria (Csuzdi \& Zicsi 2003, Valchovski 2012).

\section{Allolobophora mehadiensis boscaiui Pop, 1948}

Allolobophora mehadiensis boscaiui Pop, 1948

Serbiona mehadiensis boscaiui: Mršić 1991: 187. Šapkarev 1997: 104.

Allolobophora mehadiensis voivodinensis Šapkarev, 1989: 40. Stojanović \& Milutinović 2014: 308. (Szederjesi et al. 2014b)

Serbiona mehadiensis voivodinensis: Mršić 1991: 189. Šapkarev 1997: 104.

Allolobophora mehadiensis boscaiui: Szederjesi et al. 2014b: 86.

Distribution. Romania, Serbia (Mršić 1991).

\section{Allolobophora paratuleskovi Šapkarev, 1975}

Allolobophora paratuleskovi Šapkarev, 1975b: 55. Trakić et al. 2016: 256. Stojanović et al. 2017a: 180.

Serbiona paratuleskovi: Mršić 1991: 206. Šapkarev 1997: 104.

Distribution. Serbia (Mršić 1991).

\section{Allolobophora pyrenaicoides (Šapkarev, 1977)}

Eophila pyrenaicoides Šapkarev, 1977a: 75.

Italobalkaniona pyrenaicoides: Mršić 1991: 162. Šapkarev 1997: 103.

Allolobophora pyrenaicoides: Csuzdi 2012. Trakić et al. 2016: 256.

Distribution. Macedonia (Mršić 1991).

\section{Allolobopphora robusta robusta Rosa, 1895}

Allolobophora robusta Rosa, 1895: 2.

Serbiona robusta robusta: Mršić 1991: 162. Šapkarev 1997: 104. Stojanović \& Karaman 2007: 23. Valchovski 2012: 99. Stojanović et al. 2013: 639.

Allolobophora robusta robusta: Szederjesi 2013a: 62. Stojanović et al. 2012: 9. Stojanović \& Milutinović 2014: 308.

Distribution. Romania, Bulgaria, Serbia (Mršić 1991).
Allolobophora robusta spasenijakaramani (Blakemore, 2004)

Allolobophora robusta serbica Karaman, 1983: 52. Serbiona robusta serbica: Mršić 1991: 162. Šapkarev 1997: 104.

Serbiona spasenijakaramani Blakemore, 2004: 78. (nom.nov.)

Allolobophora robusta spasenijakaramani: Csuzdi 2012. Stojanović et al. 2013: 639.

Allolobophora spasenijakaramani: Trakić et al. 2016: 257. Stojanović et al. 2017a: 181.

Distribution. Serbia (Mršić 1991).

\section{Allolobophora ruzsai Szederjesi, 2014}

Allolobophora ruzsai Szederjesi, 2014: 48. Trakić et al. 2016: 256.

Distribution. Montenegro (Szederjesi 2014).

\section{Allolobophora serbica (Šapkarev, 1977)}

Eophila serbica Šapkarev, 1977a: 93.

Serbiona serbica: Mršić 1991: 162. Šapkarev 1997: 104.

Allolobophora serbica: Csuzdi 2012. Stojanović et al. 2017a: 181.

Distribution. Serbia (Mršić 1991).

\section{Allolobophora speciosa Mršić \& Šapkarev, 1987}

Eophila speciosa Mršić \& Šapkarev, 1987: 69.

Serbiona speciosa: Mršić 1991: 202. Šapkarev 1997: 104.

Allolobophora speciosa: Csuzdi 2012. Trakić et al. 2016: 257.

Distribution. Serbia (Mršić 1991).

\section{Allolobophora stankovici (Šapkarev, 1971)}

Allolobophora januaeargenti stankovici Šapkarev, 1971: 152

Italobalkaniona stankovici: Mršić 1991: 165. Šapkarev 1997: 103.

Allolobophora stankovici: Csuzdi 2012. Trakić et al. 2016: 257.

Distribution. Macedonia (Mršić 1991). 


\section{Allolobophora strumicae Šapkarev, 1973}

Allolobophora dofleini strumicae Šapkarev, 1973: 44.

Serbiona strumicae: Mršić 1991: 183. Šapkarev 1997: 104.

Allolobophora strumicae: Csuzdi 2012. Trakić et al. 2016: 257.

Distribution. Macedonia (Mršić 1991).

\section{Allolobophora sturanyi sturanyi Rosa, 1895}

Allolobophora sturanyi Rosa, 1895: 5.

Karpatodinariona sturanyi: Mršić 1991: 250. Stojanović et al. 2008: 59.

Allolobophora sturanyi sturanyi: Hackenberger Kutuzović \& Hackenberger Kutuzović 2013: 5. Stojanović \& Milutinović 2013: 148. Szederjesi 2013a: 62.

Distribution. Croatia, Serbia, Bosnia-Herzegovina, Montenegro (Mršić 1991).

\section{Allolobophora sturanyi dacica (Pop, 1938)}

Eophila dacica Pop, 1938: 142.

Karpatodinariona dacica: Mršić 1991: 246.

Allolobophora sturanyi dacica: Csuzdi \& Pop 2008: 26.

Distribution. Hungary, Romania, Moldova, Serbia, Bosnia-Herzegovina and Croatia (Csuzdi \& Zicsi 2003).

\section{Allolobophora treskavicensis (Mršić, 1991)}

Italobalkaniona treskavicensis Mršić, 1991: 165. Šapkarev 1997: 103.

Allolobophora treskavicensis: Csuzdi 2012. Trakić et al. 2016: 257.

Distribution. Macedonia (Mršić 1991).

\section{Allolobophora tuleskovi (Černosvitov, 1937)}

Eophila tuleskovi Černosvitov, 1937a: 87.

Serbiona tuleskovi: Mršić 1991: 205. Šapkarev 1997: 104.

Serbiona tuleshkovi: Valchovski 2012: 99. (sic!)

Allolobophora tuleskovi: Csuzdi 2012. Stojanović et al. 2012: 9. Trakić et al. 2016: 258.

Distribution. Bulgaria (Valchovski 2012).

\section{Allolobophora yugoslavica (Šapkarev, 1977)}

Eophila yugoslavica Šapkarev, 1977a: 89.

Serbiona yugoslavica: Mršić 1991: 196. Šapkarev 1997: 104.

Allolobophora yugoslavica: Csuzdi 2012. Trakić et al. 2016: 258 .

Distribution. Serbia (Mršić 1991).

\section{Allolobophora zicsi Šapkarev, 1975}

Allolobophora zicsi Šapkarev, 1975c: 44. Trakić et al. 2016: 258.

non Allolobophora zicsii Bouché, 1972: 424.

Allolobophora orahovacensis Reynolds \& Cook 1976: 148. (nom. nov.)

Allolobophora sapkarevi Easton, 1983: 486. (nom. nov)

Italobalkaniona zicsii: Mršić 1991: 168. Šapkarev 1997: 103. (sic!)

\section{Distribution. Serbia (Mršić 1991).}

Remarks. There are some confusion in the literature regarding the name Allolobophora zicsi Šapkarev, 1975. Bouché (1972) described a species Allolobophora (s.l.) zicsii from France. Here the epithet is clearly a genitive and is formed correctly from a modern personal name (Zicsi) (ICZN Art. 31.1.2). Later, Šapkarev (1975) described Allolobophora zicsi, here the epithet is clearly a noon in apposition and also correct according to ICZN (Art. 31.1.1) and does not fall in homonymy (ICZN Art. 31.1.3 and example). Therefore the names A. orahovacensis Reynolds and Cook, 1976 and the later A. sapkarevi Easton, 1983 are unnecessary replacement names.

\section{Genus Aporrectodea Örley, 1885}

\section{Aporrectodea caliginosa caliginosa Savigny, 1826}

Enterion caliginosum Savigny, 1826: 180.

Allolobophora (Allolobophora) caliginosa: Rosa 1893a: 7.

Allolobophora caliginosa caliginosa: Karaman \& Stojanović 1995: 141.

Aporrectodea (Aporrectodea) caliginosa caliginosa: Mršić 1991: 321.

Aporrectodea caliginosa: Csuzdi \& Pavlíček 2005a: 71.; 2005b: 88. Pavlíček \& Csuzdi 2006a: 183.; 2006b: S114.; 2008: 193.; 2017: 592. Csuzdi et al. 2006: 4. (part.). Misırlıŏglu 
2009: 22. (part.). Dhora 2010: 82. Stojanović et al. 2013: 639.; 2017a: 181. Hackenberger Kutuzović \& Hackenberger Kutuzović 2013: 5. Stojanović \& Milutinović 2013: 149.; 2014: 308. Szederjesi et al. 2013a: 392.; 2013b: 201.; 2014a: 556 (part.). Szederjesi \& Csuzdi 2015: 111.

Aporrectodea caliginosa caliginosa: Pavlíček et al. 2003: 456. Stojanović \& Karaman 2003b: 56. Stojanović et al. 2012: 9. Valchovski 2012: 88.; 2014: 2. Szederjesi 2014: 49. Szederjesi et al. 2017a: 59.

Distribution. Widely distirbuted peregrine species (Csuzdi \& Zicsi 2003).

\section{Aporrectodea caliginosa trapezoides (Dugès, 1828)}

Lumbricus trapezoides Dugès, 1828: 289.

Aporrectodea (Aporrectodea) caliginosa trapezoides: Mršić 1991: 328.

Aporrectodea caliginosa trapezoides: Šapkarev 2001: 111. Pavlíček et al. 2003: 456. Valchovski 2012: 88.; 2014: 3. Szederjesi et al. 2016.; 2017a: 60.

Aporrectodea trapezoides: Stojanović et al. 2012: 9.; 2013: 639.; 2017a: 182. Hackenberger Kutuzović \& Hackenberger Kutuzović 2013: 8. Stojanović \& Milutinović 2013: 151.; 2014: 308.

Aporrectodea caliginosa (part.): Csuzdi et al. 2006: 4.; 2007: 348. Szederjesi et al. 2013b: 91.; 2014a: 556 .

Distribution. Widely distributed peregrine species (Csuzdi \& Zicsi 2003).

\section{Aporrectodea cemernicensis Mršić, 1991}

Aporrectodea (Aporrectodea) cemernicensis Mršić, 1991: 284. Šapkarev 1997: 104.

Aporrectodea cemernicensis: Szederjesi 2013a: 63. Trakić et al. 2016: 258.

Distribution. Bosnia-Herzegovina, Serbia (Szederjesi 2013a).

\section{Aporrectodea dinarica (Mršić, 1987)}

Meroandriella dinarica Mršić, 1987a: 2.; 1991: 340. Šapkarev 1997: 104.
Aporrectodea dinarica: Csuzdi 2012. Trakić et al. 2016: 258.

Distribution. Slovenia (Mršić 1991).

\section{Aporrectodea dubiosa (Örley, 1881)}

Criodrilus dubiosus Örley, 1881: 603.

Allolobophora dubiosa: Šapkarev 2002: 296.

Aporrectodea (Aporrectodea) dubiosa dubiosa: Mršić 1991: 334.

Aporrectodea dubiosa dubiosa: Csuzdi et al. 2006: 4. Misırlığlu et al. 2008: 79. Misırlığlu 2009: 22.

Aporrectodea dubiosa: Stojanović et al. 2012: 9. Valchovski 2012: 88. Hackenberger Kutuzović \& Hackenberger Kutuzović 2013: 6. Stojanović \& Milutinović 2014: 308.

Distribution. From Slovakia to the shore of the Black Sea, and its eastern shore (Csuzdi \& Zicsi 2003).

\section{Aporrectodea georgii (Michaelsen, 1890)}

Allolobophora georgii Michaelsen, 1890: 3.

Aporrectodea (Aporrectodea) georgii: Mršić 1991: 315.

Aporrectodea georgii: Dhora 2010: 82. Milutinović et al. 2010: 629. Stojanović et al. 2012: 9.; 2017a: 181. Valchovski 2012: 89. Szederjesi \& Csuzdi 2012b: 262. Hackenberger Kutuzović \& Hackenberger Kutuzović 2013: 6. Stojanović \& Milutinović 2013: 149. Szederjesi 2013a: 63.; Szederjesi et al. 2017a: 60.

Distribution. widely distributed Atlanto-Mediterranean species (Csuzdi \& Zicsi 2003).

\section{Aporrectodea handlirschi handlirschi (Rosa, 1897)}

Allolobophora handlirschi Rosa, 1897: 3.

Eiseniona handlirschi rhenani: Misırlığlu 2009: 23.

Aporrectodea (Aporrectodea) handlirschi: Mršić 1991: 292.

Aporrectodea handlirschi handlirschi: Csuzdi et al. 2006: 4. Misırlığlu et al. 2008: 79. Stojanović et al. 2008: 59. Szederjesi et al. 2017a: 60.

Aporrectodea handlirschi: Valchovski 2012: 89. Stojanović et al. 2012: 9.; 2013: 639.; 2017a: 
181. Hackenberger Kutuzović \& Hackenberger Kutuzović 2013: 6. Stojanović \& Milutinović 2013: 150. Szederjesi 2013a: 63.

Distribution. from Italy and Poland to the Caucasus (Csuzdi \& Zicsi 2003).

\section{Aporrectodea handlirschi mahnerti (Zicsi, 1973)}

Allolobophora handlirschi mahnerti Zicsi, 1973: 230. Csuzdi et al. 2006: 6. Misırlığlu et al. 2008: 79. Misırlığlu 2009: 22. Szederjesi et al. 2014a: 557.

Distribution. Turkey (Csuzdi et al. 2006).

\section{Aporrectodea jassyensis (Michaelsen, 1891)}

Allolobophora jassyensis Michaelsen, 1891: 15.

Allolobophora (Allolobophora) jassyensis: Rosa 1893a: 8.

Aporrectodea (Aporrectodea) jassyensis: Mršić 1991: 316. Šapkarev 1997: 104.

Aporrectodea jassyensis jassyensis: Csuzdi et al. 2006: 6. Misırlığlu 2009: 22. Valchovski 2012: 89.; 2014: 3.

Aporrectodea jassyensis: Pavlíček et al. 2003: 456. Csuzdi \& Pavlíček 2005a: 72.; 2005b: 89. Csuzdi et al. 2007: 349. Misırlığlu et al. 2008: 79. Dhora 2010: 82. Stojanović et al. 2012: 9.; 2013: 639.; 2017a: 181. Hackenberger Kutuzović \& Hackenberger Kutuzović 2013: 7. Stojanović \& Milutinović 2013: 150.; 2014: 308. Szederjesi 2013b: 77.; Szederjesi et al. 2013b: 202.; 2014a: 557.; 2017a: 61. Valchovski \& Szederjesi 2016: 357. Pavlíček \& Csuzdi 2017: 593.

Distribution. Widely distributed Trans-Aegean species (Csuzdi \& Zicsi 2003).

\section{Aporrectodea macvensis (Šapkarev, 2002)}

Allolobophora macvensis Šapkarev, 2002: 299.

Aporrectodea (Aporrectodea) macvensis: Mršić 1991: 294. Šapkarev 1997: 104.

Aporrectodea macvensis: Stojanović \& Milutinović 2014: 308. Trakić et al. 2016: 258. Stojanović et al. 2017a: 181.

Distribution. Serbia (Mršić 1991).

\section{Aporrectodea longa (Ude, 1885)}

Allolobophora longa Ude, 1885: 136.

Aporrectodea (Aporrectodea) longa: Mršić 1991: 330. Stojanović et al. 2012: 9. Valchovski 2012: 90. Szederjesi et al. 2017a: 61.

Distribution. Widely distributed peregrine species of Atlantic origin (Csuzdi \& Zicsi 2003).

\section{Aporrectodea rosea (Savigny, 1826)}

Enterion roseum Savigny, 1826: 182.

Aporrectodea (Aporrectodea) rosea rosea: Mršić 1991: 296.

Aporrectodea (Aporrectodea) rosea balcanica: Mršić 1991: 303. Šapkarev 1997: 104.

Aporrectodea (Aporrectodea) rosea bimastoides: Mršić 1991: 304.

Aporrectodea rosea: Šapkarev 2001: 111. Pavlíček et al. 2003: 456. Stojanović \& Karaman 2003b: 55.; 2005a: 128. Csuzdi \& Pavlíček 2005a: 72.; 2005b: 89. Csuzdi et al. 2006: 6. Pavlíček \& Csuzdi 2006a: 184.; 2006b: S114.; 2008: 193.; 2017: 592. Csuzdi et al. 2007: 349. Misırlığlu 2009: 22. Dhora 2010: 82. Szederjesi \& Csuzdi 2012b: 262. Stojanović et al. 2012:9.; 2013: 639.; 2017a: 182. Hackenberger Kutuzović \& Hackenberger Kutuzović 2013: 7. Szederjesi 2013a: 64. Stojanović \& Milutinović 2013: 150.; 2014: 308. Szederjesi et al. 2013a: 392.; 2013b: 202.; 2014a: 557.; 2017a: 61. Valchovski \& Szederjesi 2016: 357.

Allolobophora rosea balcanica: Šapkarev 2002: 296.

Aporrectodea rosea rosea: Valchovski 2012: 90.; 2014: 3

Distribution. Widely distributed peregrine species (Csuzdi \& Zicsi 2003).

\section{Aporrectodea sineporis (Omodeo, 1952)}

Eiseniella balcanica sine-poris Omodeo, 1952: 31.

Aporrectodea (Aporrectodea) sineporis: Mršić 1991: 287.

Aporrectodea sineporis: Stojanović \& Karaman 2005b: 133. Milutinović et al. 2010: 629. Hackenberger Kutuzović \& Hackenberger Kutuzović 2013: 8. Szederjesi 2013a: 64. Stojanović et al. 2017a: 182. 
Distribution. Italy, Austria, Hungary, Slovenia, Serbia (Csuzdi \& Zicsi 2003, Stojanović \& Karaman 2005b).

\section{Aporrectodea smaragdina (Rosa, 1892)}

Allolobophora smaragdina Rosa, 1892: 1. Karaman \& Stojanović 1995: 139.

Aporrectodea (Aporrectodea) smaragdina: Mršić 1991: 308.

Aporrectodea smaragdina: Stojanović \& Karaman 2003b: 55. Szederjesi \& Csuzdi 2012b: 263.; 2015: 112. Stojanović et al. 2013: 639.; 2017a: 182. Hackenberger Kutuzović \& Hackenberger Kutuzović 2013: 8. Stojanović \& Milutinović 2013: 150. Szederjesi 2013a: 65.; 2014: 49.

Aporrectodea (Aporrectodea) smaragdinoides Šapkarev, 1989: 42.; 1997: 104. Mršić 1991: 312. (Szederjesi 2013a).

Distribution. From Italy to Serbia (Mršić 1991).

\section{Genus Bimastos Moore, 1891}

\section{Bimastos eiseni (Levinsen, 1884)}

Lumbricus eiseni Levinsen, 1884: 241.

Eisenia eiseni: Karaman \& Stojanović 2002: 224. Stojanović \& Karaman 2003b: 57.

Allolobophoridella eiseni: Mršić 1991: 255. Dhora 2010: 82. Valchovski 2012: 87. Szederjesi \& Csuzdi 2012b: 262; 2015: 111. Stojanović et al. 2012: 9.; 2013: 639. Hackenberger Kutuzović \& Hackenberger Kutuzović 2013: 5. Stojanović \& Milutinović 2013: 149. Szederjesi 2014: 49. Valchovski \& Szederjesi 2016: 356. Szederjesi et al. 2017a: 59.

Bimastos eiseni: Karaman \& Stojanović 1995: 140. Csuzdi et al. 2017: 13.

Distribution. Peregrine species of North American origin (Csuzdi et al. 2017).

\section{Bimastos parvus (Eisen, 1874)}

Allolobophora parva Eisen, 1874: 46.

Bimastos parvus: Pavlíček et al. 2003: 455. Pavlíček \& Csuzdi 2006a: 184. Szederjesi et al. 2013b: 202, Csuzdi et al. 2017: 19.
Distribution. Widely distributed peregrine species of North American origin (Pavlíček et al. 2003).

\section{Bimastos rubidus (Savigny, 1826)}

Enterion rubidum Savigny, 1826: 182.

Dendrobaena rubida rubida: Karaman \& Stojanović 1995: 140.

Dendrobaena rubida tenuis: Karaman \& Stojanović 1995: 140.

Dendrodrilus rubidus rubidus: Mršić 1991: 263. Šapkarev 2001: 111. Stojanović \& Karaman 2003b: 57.; 2005a: 130. Misirlığlu 2009: 22. Stojanović et al. 2012: 10.; 2013: 639.; 2017a: 184. Szederjesi \& Csuzdi 2012b: 269.; 2015: 112. Valchovski 2012: 93. Hackenberger Kutuzović \& Hackenberger Kutuzović 2013: 12. Stojanović \& Milutinović 2013: 154.; 2014: 308. Szederjesi 2013a: 69.; 2014: 50. Szederjesi et al. 2014a: 565.; 2017a: 66. Valchovski \& Szederjesi 2016: 357.

Dendrodrilus rubidus tenuis: Mršić 1991: 270. Šapkarev 2001: 111. Stojanović \& Karaman 2003b: 56.; 2005a: 130. Stojanović et al. 2012: 10.; 2017a: 184. Valchovski 2012: 94.

Dendrodrilus rubidus subrubicundus: Mršić 1991: 267. Šapkarev 2001: 111. Misırlıŏglu 2009: 22. Pavlíček \& Csuzdi 2008: 194.; 2017: 592. Dhora 2010: 82. Valchovski 2012: 93. Szederjesi \& Csuzdi 2012b: 269. Stojanović et al. 2012: 10.; 2013: 639.; 2017a: 184. Hackenberger Kutuzović \& Hackenberger Kutuzović 2013: 12. Stojanović \& Milutinović 2013: 154.; 2014: 308. Szederjesi 2013a: 69.; 2014: 50.; Szederjesi et al. 2016.; 2017a: 67.

Dendrodrilus rubidus subrubicunda: Karaman \& Stojanović 2002: 224.

Bimastos rubidus: Csuzdi et al. 2017: 20.

Distribution. Peregrine species of North American origin (Csuzdi et al. 2017).

\section{Genus Cernosvitovia Omodeo, 1956}

\section{Cernosvitovia biserialis (Černosvitov, 1937)}

Allolobophora biserialis Černosvitov, 1937a: 85.

Cernosvitovia (Cernosvitovia) biserialis: Mršić 1991: 142. Šapkarev 1997: 103.; 2002: 300. 
Stojanović et al. 2012: 10.; 2013: 639. Valchovski 2012: 90.

Cernosvitovia biserialis: Trakić et al. 2016: 258.

Distribution. Serbia, Bulgaria (Mršić 1991).

\section{Cernosvitovia dobrogeana (Pop, 1938)}

Octolasium dobrogeanum Pop, 1938: 148.

Cernosvitovia (Cernosvitovia) dobrogeana: Mršić 1991: 144. Stojanović et al. 2012: 10. Valchovski 2012: 90.

Cernosvitovia dobrogeana: Trakić et al. 2016: 259.

Distribution. Romania, Bulgaria (Mršić 1991).

\section{Cernosvitovia dudichi Zicsi \& Šapkarev, 1982}

Cernosvitovia dudichi Zicsi \& Šapkarev, 1982: 181.

Cernosvitovia (Zicsiona) dudichi: Mršić 1991: 149. Šapkarev 1997: 103.

Cenrosvitovia dudichi: Trakić et al. 2016: 259.

Distribution. Serbia (Mršić 1991).

\section{Cernosvitovia getica (Pop, 1947)}

Allolobophora dugesi v. getica Pop, 1947: 8.

Cernosvitovia (Zicsiona) getica: Mršić 1991: 152. Šapkarev 1997: 103.

Eophila getica: Šapkarev 2002: 302.

Cernosvitovia getica: Csuzdi \& Pop 2007: S20 (for complete synonymy). Trakić et al. 2016: 259.

Distribution. Romania, Serbia (Mršić 1991).

\section{?Cernosvitovia knazevensis (Šapkarev, 1989)}

Italobalkaniona knazevensis Šapkarev, 1989: 38.; 1997: 103. Mršić 1991: 173.

\section{Distribution. Serbia (Mršić 1991).}

Remarks. The exact position of the male pore is not stated in the original description. Šapkarev (1989) only mentions that it is invisible. Therefore, inclusion of this species to Cernosvitovia is uncertain.

\section{Cernosvitovia krainensis (Šapkarev, 2002)} 303

Eophila opisthocystis krainensis Šapkarev, 2002:
Italobalkaniona opisthocystis krainensis: Mršić 1991: 172. Šapkarev 1997: 103.

Cernosvitovia krainensis: Csuzdi 2012. Stojanović et al. 2013: 639. Trakić et al. 2016: 259.

Distribution. Romania, Serbia (Mršić 1991).

Remarks. This species was formally described in 2002 but appeared in the literature as early as 1991 (Mršić 1991: 172) referring to a manuscript in press which actually has never been published. C. krainensis differs from knazevensis only in the number and position of spermathecae (7 vs. 5 pairs in 14-20 vs. 12-16). However, C. knazevensis was presumbably described from a preadult specimen, therefore the synonymy of the two species' names as suggested by Csuzdi (2012) is possible, but requires further corroboration by examining fully adult specimens of knazevensis.

\section{Cernosvitovia munteniana Zicsi \& Pop, 1991}

Cernosvitovia munteniana Zicsi \& Pop, 1991: 125. Mršić 1992: 22. Trakić et al. 2016: 259. Valchovski \& Szederjesi 2016: 357.

Distribution. Romania, Bulgaria (Valchovski \& Szederjesi 2016).

\section{Cernosvitovia opisthocystis (Rosa, 1895)}

Allolobophora opisthocystis Rosa, 1895: 4

Italobalkaniona opisthocystis: Mršić 1991: 170. Šapkarev 1997: 103.

Cernosvitovia (Zicsiona) crnicae: Mršić 1991: 155. Šapkarev 1997: 103.

Distribution. Romania, Serbia (Mršić 1991).

\section{Cernosvitovia rebeli (Rosa, 1897)}

Allolobophora rebelii Rosa, 1897: 2.

Cernosvitovia (Cernosvitovia) rebeli: Mršić 1991: 148. Šapkarev 1997: 103.

Cernosvitovia rebeli: Dhora 2010: 82. Szederjesi \& Csuzdi 2012b: 263. Stojanović et al. 2012: 10.; 2013: 639. Valchovski 2012: 91. Szederjesi 2013b: 78. Trakić et al. 2016: 260. Szederjesi et al. 2017a: 62.

Distribution. Romania, Bulgaria, Greece, Albania (Mršić 1991). 


\section{Cernosvitovia schweigeri (Zicsi, 1973)}

Allolobophora schweigeri Zicsi, 1973: 226.

Cernosvitovia schweigeri: Csuzdi et al. 2006: 8. Misırlığlu et al. 2008: 79. Misirlığlu 2009: 22.

Cenrosvitovia (Cernosvitovia) schweigeri: Šapkarev 1997: 103.

Distribution. Turkey (Csuzdi et al. 2006).

\section{Genus Dendrobaena Eisen, 1873}

\section{Dendrobaena sp.}

Dendrobaena byblica byblica: Szederjesi et al. 2014a: 558.

Distribution. European part of Turkey (Szederjesi et al. 2017b).

Remarks. Only two characters separate this species from $D$. byblica: the number of the seminal vesicles ( $3 v s .4)$ and the position of the tubercles (1/n 25, 26-1/229 vs. 26-28).

\section{Dendrobaena alexandrii Szederjesi, Pavlíček \& Csuzdi, 2013}

Dendrobaena alexandrii Szederjesi, Pavlíček \& Csuzdi, 2013a: 392.

Distribution. Jordan (Szederjesi et al. 2013a).

\section{Dendrobaena alpina alpina (Rosa, 1884)}

Allolobophora alpina Rosa, 1884: 28.

Dendrobaena alpina alpina: Mršić 1991: 627. Šapkarev 1993: 17. Szederjesi \& Csuzdi 2012b: 264.; 2015: 112. Hackenberger Kutuzović \& Hackenberger Kutuzović 2013: 9. Stojanović \& Milutinović 2013: 151. Szederjesi 2013a: 66.; 2013b: 78. Stojanović et al. 2017a: 182. Szederjesi et al. 2017a: 62.

Dendrobaena alpina: Šapkarev 2001: 112. Stojanović \& Karaman 2005a: 129. Stojanović et al. 2012: 10. Valchovski 2012: 91.

Dendrobaena alpina mavrovensis Šapkarev, 1971: 160.; 1997: 105. Mršić 1991: 630. syn. nov.

Distribution. The Alpes, the Balkans and the Southern Carpathians (Csuzdi et al. 2011).
Remarks. Examining of two syntypes of $D$. alpina mavrovensis housed in HNHM (HNHM/ 12673) revealed that the openings of the spermathecae are in the dorsomedian line like in case of the nominal subspecies and not in setal line $d$, as Šapkarev (1971) noted it in the original description. Therefore D. alpina mavrovensis is regarded as a synonym of $D$. alpina alpina.

\section{Dendrobaena alpina armeniaca (Rosa, 1893)}

Allolobophora alpina v. armeniaca Rosa, 1893b: 431.

Dendrobaena alpina armeniaca: Csuzdi et al. 2006: 8. Misırlığlu et al. 2008: 79.. Misırlığlu 2009: 22. Szederjesi et al. 2014a: 558.

Distribution. Armenia, Turkey (Rosa 1893b, Omodeo \& Rota 1989).

\section{Dendrobaena alpina popi Šapkarev, 1971}

Dendrobaena alpina popi Šapkarev, 1971: 159.; 1993: 17.; 1997: 105. Mršić 1991: 634. Csuzdi et al. 2011: 13. Pop et al. 2012: 63. Szederjesi \& Csuzdi 2012b: 264. Szederjesi 2013a: 66.

Distribution. Romania, Bosnia-Herzegovina, Macedonia, Montenegro, Albania (Csuzdi et al. 2011, Szederjesi \& Csuzdi 2012b).

\section{Dendrobaena attemsi (Michaelsen, 1902)}

Helodrilus (Dendrobaena) attemsi Michaelsen, 1902: 47.

Dendrobaena attemsi: Mršić 1991: 604. Šapkarev 1993: 17.; 2001: 112.; 2002: 295. Stojanović \& Karaman 2005a: 129. Csuzdi et al. 2006: 8. Misırlığlu et al. 2008: 79. Misırlıŏlu 2009: 22. Valchovski 2012: 91. Szederjesi \& Csuzdi 2012b: 264.; 2015: 112. Stojanović et al. 2012:10; 2013: 639. Pop et al. 2012: 62. Hackenberger Kutuzović \& Hackenberger Kutuzović 2013: 9. Szederjesi 2013a: 66.; 2013b: 79. Szederjesi et al. 2017a: 62.

Dendrobaena attemsi attemsi: Stojanović et al. 2017a: 182.

Dendrobaena jastrebensis Mršić \& Šapkarev, 1987: 69. Mršić 1991: 583. Šapkarev 1993: 17.; 1997: 105. Stojanović \& Milutinović 2013: 152. Stojanović et al. 2013: 639.; 2017a: 183. Trakić et al. 2016: 260. 
Dendrobaena macedonica Mršić, 1991: 587. Šapkarev 1997: 105. Trakić et al. 2016: 262.

Dendrobaena vranicensis Mršić, 1991: 588. Šapkarev 1997: 105. Trakić et al. 2016: 263.

Dendrobaena grmecensis: Mršić, 1991: 593. Šapkarev 1997: 105.

Distribution. From the Pyrenees through the Balkans and Anatolia to the Caucasus (Omodeo \& Rota 1999), but it has been indroduced to several regions as well (Blakemore 2008).

Remarks. D. attemsi is morphologically highly variable. Its colour varies from overall dark red to red just at the head on dorsum. Also its tubercles are in variable position from 30-31, 1/n $32,1 / 232$, 32 therefore Csuzdi (2012) suggested to synonimize grmecensis, jastrebensis, macedonica and vranicensis to D. attemsi.

D. attemsi is easily identified apart from the clitellar organs by its last pair of hearts in segment 9 or 10 and the regularly alternating nephridiopores. Unfortunately neither of these two characters were mentioned in the description of the four species synonymized.

\section{Dendrobaena balcanica (Černosvitov, 1937)}

Eisenia veneta var. balcanica Černosvitov, 1937a: 81.

Dendrobaena balcanica: Mršić 1991: 620. Šapkarev 1997: 105. Stojanović et al. 2012: 10.Valchovski 2012: 92. Trakić et al. 2016: 260. Szederjesi et al. 2017a: 62.

Distribution. Bulgaria, Greece (Szederjesi \& Csuzdi 2012a).

\section{Dendrobaena bokakotorensis Šapkarev, 1975}

Dendrobaena bokakotorensis Šapkarev, 1975c: 4.; 1993: 17.; 1997: 105. Mršić 1991: 565. Stojanović \& Milutinović 2013: 151. Trakić et al. 2016: 260. Szederjesi et al. 2017a: 63.

Distribution. Croatia, Montenegro, Greece (Mršić 1991).

\section{Dendrobaena bosniaca Mršić, 1988}

Dendrobaena bosniaca Mršić, 1988: 14. 1991: 586. Šapkarev 1997: 105. rakić et al. 2016: 260.

Distribution. Bosnia-Herzegovina (Mršić, 1991, Trakić et al. 2016).

\section{Dendrobaena bruna Omodeo \& Rota, 1989}

Dendrobaena bruna Omodeo \& Rota, 1989: 196. Csuzdi et al. 2006: 8. Misırlığlu et al. 2008: 79. Misırlıoğlu 2009: 22.

Distribution. Turkey (Csuzdi et al. 2006).

\section{Dendrobaena byblica byblica (Rosa, 1893)}

Allolobophora (Dendrobaena) byblica Rosa, 1893a: 4.

Dendrobaena byblica: Mršić 1991: 566. Šapkarev 1993: 17.; 2001: 113. Karaman \& Stojanović 1995: 140. Pavlíček et al. 2003: 456. Stojanović \& Karaman 2003b: 56.; 2005a: 129. Csuzdi \& Pavlíček 2005a: 72. Pavlíček \& Csuzdi 2006b: S114; 2008: 193; 2017: 598. Misırlığlu et al. 2008: 79. Misirlığlu 2009: 22. Dhora 2010: 82. Stojanović et al. 2012: 10.; 2013: 639.; 2017a: 183. Valchovski 2012: 92. Szederjesi et al. 2017b: in press.

Dendrobaena byblica byblica: Csuzdi et al. 2006: 8.; 2007: 350. Szederjesi \& Csuzdi 2012b: 265.; 2015: 112. Hackenberger Kutuzović \& Hackenberger Kutuzović 2013: 10. Stojanović \& Milutinović 2013: 151. Szederjesi 2013a: 67.; 2013b: 79.; 2014: 49.; Szederjesi et al. 2013a: 394.; 2013b: 203.; 2014a: 558.; 2017a: 63.

Distribution. widely distributed in the Mediterranean (Pavlíček \& Csuzdi 2017).

\section{Dendrobaena cevdeti Szederjesi, Pavlíček, Coş- kun \& Csuzdi, 2014}

Dendrobaena cevdeti Szederjesi, Pavlíček, Coşkun \& Csuzdi 2014: 561.

Distribution. Turkey (Szederjesi et al. 2014a). 


\section{Dendrobaena cognettii (Michaelsen, 1903)}

Helodrilus cognettii Michaelsen, 1903: 140.

Dendrobaena cognettii: Hackenberger Kutuzović \& Hackenberger Kutuzović 2013: 10. Szederjesi 2013a: 67. Szederjesi et al. 2014a: 558.; 2017a: 63.

Dendrobaena pygmaea: Mršić 1991: 643.

Distribution. From Western Europe through the Balkans to Turkey (Csuzdi \& Zicsi 2003).

\section{Dendrobaena decipiens (Michaelsen, 1910)}

Helodrilus alpinus decipiens Michaelsen, 1910b: 33.

Dendrobaena decipiens: Csuzdi et al. 2006: 10. Misırlıoğlu et al. 2008: 79. Misırlıoğlu 2009: 22.

Distribution. Turkey, Georgia (Omodeo \& Rota 1989).

\section{Dendrobaena depressa (Rosa, 1893)}

Allolobophora platyura depressa Rosa, 1893b: 543.

Fitzingeria platyura depressa: Mršić 1991: 543. Šapkarev 1993: 17. Stojanović et al. 2012: 11. Valchovski 2012: 95. Hackenberger Kutuzović \& Hackenberger Kutuzović 2013: 13. Szederjesi 2013a: 70. Stojanović \& Milutinović 2014: 308.

Fitzingeria viminiana: Mršić 1991: 539. Šapkarev 1993: 17.; 1997: 104. (Csuzdi \& Zicsi 2003)

Dendrobaena depressa: Szederjesi et al. 2017b: in press.

Distribution. From Austria through the Carpathian Basin to Macedonia and Bulgaria (Csuzdi \& Zicsi 2003).

\section{Dendrobaena epirotica Zicsi \& Michalis, 1993}

Dendrobaena epirotica Zicsi \& Michalis, 1993: 306. Trakić et al. 2016: 261. Szederjesi et al. 2017a: 64.

Distribution. Greece (Zicsi \& Michalis 1993).

\section{Dendrobaena feheri Szederjesi \& Csuzdi, 2017}

Dendrobaena feheri Szederjesi \& Csuzdi, 2017 in: Szederjesi et al. 2017b: in press.
Dendrobaena ganglbaeri (part.): Szederjesi \& Csuzdi 2012b: 265.

Distribution. Albania (Szederjesi et al. 2017b).

\section{Dendrobaena fridericae fridericae Omodeo \& Rota, 1989}

Dendrobaena fridericae Omodeo \& Rota, 1989: 186. Csuzdi et al. 2006: 10. Misırlığlu et al. 2008: 79. Misırlığlu 2009: 22.

Distribution. Turkey (Csuzdi et al. 2006).

\section{Dendrobaena fridericae uludagi Omodeo \& Rota, 1991}

Dendrobaena fridericae uludagi Omodeo \& Rota, 1991: 179. Csuzdi et al. 2006: 10. Misırlığlu et al. 2008: 79. Misirlığlu 2009: 22.

Distribution. Turkey (Csuzdi et al. 2006).

\section{Dendrobaena ganglbaueri (Rosa, 1894)}

Allolobophora ganglbaueri Rosa, 1894: 1. Dendrobaena byblica: Mršić 1991: 566. (part.)

Dendrobaena ganglbaueri: Szederjesi \& Csuzdi 2012b: 265. Hackenberger Kutuzović \& Hackenberger Kutuzović 2013: 10. Szederjesi 2013a: 67. Szederjesi et al. 2017b. in press.

Distribution. Hungary, Slovenia, Croatia, Bosnia-Herzegovina, Serbia (Csuzdi \& Zicsi 2003).

\section{Dendrobaena hamzalensis Mršić, 1991}

Dendrobaena hamzalensis Mršić, 1991: 639. Trakić et al. 2016: 261.

Distribution. Macedonia (Mršić 1991).

\section{Dendrobaena hauseri Zicsi, 1973}

Dendrobaena hauseri Zicsi, 1973: 222. Pavlíček et al. 2003: 456. Csuzdi et al. 2006: 10. Misırlığlu et al. 2008: 79. Misırlığlu 2009: 22. Szederjesi et al. 2013b: 203.

Distribution. Turkey, Israel (Pavlíček et al. 2003). 


\section{Dendrobaena hortensis (Michaelsen, 1890)}

Allolobophora subrubicunda var. hortensis Michaelsen, 1890: 15.

Dendrobaena hortensis: Mršić 1991: 622. Šapkarev 1993: 17.; 2002: 296. Pavlíček et al. 2003: 456. Csuzdi et al. 2006: 10. Misirlığlu 2009: 22. Dhora 2010: 82. Stojanović et al. 2012:10.; 2013: 639.; 2017a: 183. Szederjesi 2013b: 79.; 2015: 146.; 2016. Valchovski 2012: 92. Szederjesi et al. 2014a: 558.; 2017a: 64.

Dendrobaena pseudohortensis Šapkarev, 1977b: 37.; 1993: 17.; 1997: 105. Mršić 1991: 625. Trakić et al. 2016: 262.

Dendrobaena slovenica Mršić, 1991: 640. Šapkarev 1997: 105.

Dendrobaena veneta ochridana: Šapkarev 1993: 17.

Dendrobaena ochridana: Šapkarev 1997: 105.

Distribution. Widely distributed peregrine species (Csuzdi \& Zicsi 2003).

Remarks. D. hortensis is a highly variable peregrine species originally described with three pairs of vesicles in $9,11,12$. The morphologically similar species (D. v. ochridana Šapkarev, 1993 and $D$. slovenica Mršić, 1991) described with two pairs of vesicles in 11, 12 were put in synonymy by Csuzdi (2012). D. pseudohortensis with 3-4 pairs of vesicles was synonymized by Csuzdi \& Zicsi (2003).

\section{Dendrobaena hrabei (Černosvitov, 1934)}

Eisenia veneta var. hrabei Černosvitov, 1934a: 72.

Dendrobaena hrabei: Mršić 1991: 631. Šapkarev 1997: 105. Stojanović et al. 2012: 10.Valchovski 2012: 92. Szederjesi 2013a: 68. Trakić et al. 2016: 261. Szederjesi et al. 2017a: 64.

Distribution. Bulgaria, Greece, Macedonia (Szederjesi et al. 2017a).

\section{Dendrobaena illyrica (Cognetti, 1906)}

Helodrilus (Dendrobaena) illyricus Cognetti, 1906: 1.

Dendrobaena illyrica: Mršić 1991: 599. Stojanović et al. 2013: 639. Hackenberger Kutuzović \& Hackenberger Kutuzović 2013: 11.
Distribution. Croatia, Bosnia-Herzegovina, Macedonia, Montenegro, Serbia (Hackenberger Kutuzović \& Hackenberger Kutuzović 2013).

\section{Dendrobaena jahorensis Mršić, 1991}

Dendrobaena jahorensis Mršić, 1991: 585. Šapkarev 1997: 105. Trakić et al. 2016: 261.

Distribution. Bosnia-Herzegovina (Mršić 1991).

\section{Dendrobaena kervillei (Michaelsen, 1910)}

Helodrilus (Eisenia) venetus var. kervillei Michaelsen, 1910a: 166.

Dendrobaena kervillei: Csuzdi \& Pavlíček 2002: 110. Pavlíček et al. 2003: 456. Szederjesi et al. 2013b: 203.

Distribution. Israel, Lebanon, Syria (Pavlíček et al. 2003).

\section{Dendrobaena kozuvensis Šapkarev, 1971}

Allolobophora kozuvensis Šapkarev, 1971: 155.

Dendrobaena kozuvensis: Mršić 1991: 564. Šapkarev 1993: 17.; 1997: 105. Stojanović \& Milutinović 2013: 152. Trakić et al. 2016: 261.

Distribution. Macedonia, Montenegro, Serbia (Mršić 1991).

\section{Dendrobaena loebli (Zicsi, 1985)}

Fitzingeria loebli Zicsi, 1985a: 330. Csuzdi et al. 2006: 20. Szederjesi et al. 2017a: 69.

Fitzingeria loebeli: Šapkarev 1997: 105. (sic!)

Fitzingeria loeblii: Mısırlığlu et al. 2008: 80. Misırlıoğlu 2009: 22. (sic!)

Dendrobaena loebli: Szederjesi et al. 2017b: in press.

Distribution. Turkey, Greece (Szederjesi \& Csuzdi 2012a).

\section{Dendrobaena luraensis Szederjesi \& Csuzdi, 2012}

Dendrobaena luraensis Szederjesi \& Csuzdi, 2012b: 266. Szederjesi 2014: 50. Trakić et al. 2016: 261.

Distribution. Albania, Montenegro (Szederjesi 2014) 


\section{Dendrobaena mahnerti Zicsi, 1974}

Dendrobaena mahnerti Zicsi, 1974: 449. Mršić 1991: 645. Šapkarev 1997: 105. Trakić et al. 2016: 262. Szederjesi et al. 2017a: 64.

Distribution. Greece (Mršić 1991).

Dendrobaena mahunkai Csuzdi, Pavlíček \& Misırlığglu, 2007

Dendrobaena mahunkai Csuzdi, Pavlíček \& Misırlığlu, 2007: 351. Misırlığlu et al. 2008: 79. Misırlığlu 2009: 22.

Distribution. Turkey (Hatay) (Csuzdi et al. 2007).

\section{Dendrobaena michalisi Karaman, 1972}

Dendrobaena michalisi Karaman, 1972b: 112. Mršić 1991: 636. Šapkarev 1997: 105. Trakić et al. 2016: 262. Szederjesi et al. 2017a: 64.

Distribution. Greece (Mršić 1991).

\section{Dendrobaena montana (Michaelsen, 1910)}

Helodrilus veneta montana Michaelsen, 1910b: 30.

Dendrobaena montana: Csuzdi et al. 2006: 10. Misırlığlu et al. 2008: 79. Misırlığlu 2009: 22.

Distribution. Turkey (Csuzdi et al. 2006).

\section{Dendrobaena montenegrina Mršić, 1988}

Dendrobaena montenegrina Mršić, 1988: 19.; 1991: 595. Šapkarev 1997: 105. Stojanović \& Milutinović 2013: 153. Trakić et al. 2016: 262.

Distribution. Montenegro (Mršić 1991).

\section{Dendrobaena negevis Csuzdi \& Pavlíček, 1999}

Dendrobaena negevis Csuzdi \& Pavlíček, 1999: 482. Pavlíček et al. 2003: 456. Pavlíček \& Csuzdi 2006a: 184. Szederjesi et al. 2013a: 394.; 2013b: 203.

Distribution. Israel, Jordan (Pavlíček et al. 2003).

\section{Dendrobaena nevoi Csuzdi \& Pavlíček, 1999}

Dendrobaena nevoi Csuzdi \& Pavlíček, 1999: 480. Pavlíček et al. 2003: 456. Szederjesi et al. 2013b: 204.

Distribution. Israel (Pavlíček et al. 2003).

\section{Dendrobaena nivalis Omodeo \& Rota, 1989}

Dendrobaena nivalis Omodeo \& Rota, 1989: 190. Csuzdi et al. 2006: 11. Misırlığlu et al. 2008: 79. Misırlıoğlu 2009: 22. Szederjesi et al. 2014a: 559.

Distribution. Turkey (Csuzdi et al. 2006).

\section{Dendrobaena octaedra Savigny, 1826}

Enterion octaedrum Savigny, 1826: 183.

Dendrobaena octaedra: Mršić 1991: 607. Šapkarev 1993: 17. Karaman \& Stojanović 1995: 139. Stojanović \& Karaman 2003b: 55.; 2005a: 130. Stojanović et al. 2012: 10.; 2013: 639.; 2017a: 183. Valchovski 2012: 93. Hackenberger Kutuzović \& Hackenberger Kutuzović 2013: 11. Stojanović \& Milutinović 2013: 153.; 2014: 308. Szederjesi 2013a: 68.; 2013b: 79.; 2014: 50. Szederjesi et al. 2017a: 65.

Distribution. Widely distributed peregrine species (Csuzdi \& Zicsi 2003).

\section{Dendrobaena olympiaca (Michaelsen, 1902)}

Helodrilus ganglbaueri olympiaca Michaelsen, 1902: 45.

Dendrobaena byblica: Mršić 1991: 566. (part.)

Dendrobaena byblica olympiaca: Szederjesi \& Csuzdi 2012a: 32. Trakić et al. 2016: 260. Szederjesi et al. 2017a: 63.

Dendrobaena olympiaca: Szederjesi et al. 2017b: in press.

Distribution. Greece (Szederjesi 2015).

\section{Dendrobaena olympica Černositov, 1938}

Eisenia olympica Černositov, 1938b: 193.

Dendrobaena olympica: Mršić 1991: 637. Šapkarev 1997: 105. Trakić et al. 2016: 262. Szederjesi et al. 2017a: 65. 
Dendrobaena olimpica: Šapkarev 1993: 17.

Dendrobaena olympica peristerica Šapkarev, 2001: 113.; 1997: 105. syn. nov.

Distribution. Greece, Macedonia (Mršić 1991).

Remarks. Dendrobaena olympica peristerica described by Šapkarev (2001) differs from the nominal subspecies only in the position of the dorsal pores, the body size and the setal arrangement. However, these characters usually show some degree of variation, therefore $D$. olympica peristerica is regarded as a synonym of D. olympica olympica.

\section{Dendrobaena omodeoi Csuzdi, Pavlíček \& Ml- sirlığlu, 2007}

Dendrobaena omodeoi Csuzdi, Pavlíček \& Misırlığlu, 2007: 352. Misirlığlu et al. 2008: 79. Misırlıŏlu 2009: 22. Szederjesi et al. 2014a: 559.

Distribution. Turkey (Hatay) (Csuzdi et al. 2007).

\section{Dendobaena orientalis orientalis Černositov, 1940}

Dendobaena orientalis Černositov, 1940: 444. Pavlíček et al. 2003: 456. Csuzdi et al. 2006: 11.; 2007: 350. Misırlığlu et al. 2008: 79. Szederjesi et al. 2013b: 204.; 2014a: 559.

Distribution. Turkey, Israel, Lebanon (Pavlíček et al. 2003).

\section{Dendrobaena orientalis karak Csuzdi \& Pavlí- ček, 2005}

Dendrobaena orientalis karak Csuzdi \& Pavlíček, 2005a: 73 . 2005a).

Distribution. Jordan (Csuzdi \& Pavlíček

\section{Dendrobaena orientaloides (Zicsi, 1985)}

Dendrobaena alpina orientaliodes Zicsi, 1985a: 326.
Dendrobaena orientaloides: Csuzdi et al. 2006: 11. Misırlığlu et al. 2008: 79. Misırlığlu 2009: 22. Szederjesi et al. 2014a: 559.

Distribution. Turkey (Csuzdi et al. 2006).

\section{Dendrobaena pantaleonis (Chinaglia, 1913)}

Helodrilus (Bimastus) pantaleonis Chinaglia, 1913: 5.

Dendrobaena pantaleonis pantaleonis: Szederjesi \& Csuzdi 2012b: 268.

Dendrobaena pantaleonis eutypica Omodeo \& Rota, 1989: 184. Csuzdi et al. 2006: 11. Misirlığlu et al. 2008: 80. Misırlığlu 2009: 22.

Dendrobaena pantaleonis balagnensis: Mršić 1991: 642. Šapkarev 1997: 105.

Dendrobaena pantaleonis: Pavlíček \& Csuzdi 2006b: S114.; 2017: 595. Szederjesi et al. 2017a: 65 .

Distribution. France, Italy, Albania, Greece, Turkey, Cyprus (Szederjesi et al. 2017a).

\section{Dendrobaena pentheri (Rosa, 1905)}

Allolobophora (Notogama) pentheri Rosa, 1905: 1.

Dendrobaena pentheri: Csuzdi et al. 2006: 12. Pavlíček \& Csuzdi 2006b: S114; 2008: 193; 2017: 596. Csuzdi et al. 2007: 350. Misırlığlu et al. 2008: 80. Misırlığlu 2009: 22. Szederjesi et al. 2014a: 560.; 2016.; 2017a: 65.

Dendrobaena aegea (Cognetti, 1913): Mršić 1991: 647. Šapkarev 1997: 105. (Szederjesi 2015).

Distribution. Greece (Rhodes), Turkey, Georgia, Azerbaijan, Armenia, Iran, Cyprus (Szederjesi et al. 2017a).

\section{Dendrobaena persimilis Omodeo \& Rota, 1989}

Dendrobaena persimilis Omodeo \& Rota, 1989: 193. Csuzdi et al. 2006: 12. Misırlıoğlu et al. 2008: 80. Misırlığlu 2009: 22.

Distribution. Turkey (Csuzdi et al. 2006).

\section{Dendrobaena perula Omodeo \& Rota, 1989}

Dendrobaena perula Omodeo \& Rota, 1989: 195. Csuzdi et al. 2006: 12. Misırlığlu et al. 2008: 80. Misırlıoğlu 2009: 22. 
Distribution. Turkey (Csuzdi et al. 2006).

\section{Dendrobaena pindonensis Zicsi \& Michalis, 1993}

Dendrobaena pindonensis Zicsi \& Michalis, 1993: 307. Trakić et al. 2016: 262. Szederjesi et al. 2017a: 66.

Distribution. Greece (Zicsi \& Michalis 1993).

\section{Dendrobaena platyura (Fitzinger, 1833)}

Enterion platyurum Fitzinger, 1833: 533.

Fitzingeria platyura platyura: Mršić 1991: 542. Šapkarev 1993: 17. Stojanović \& Karaman 2005a: 130. Szederjesi 2013a: 70. Stojanović et al. 2017a: 185.

Dendrobaena platyura: Szederjesi et al. 2017b: in press.

Distribution. Austria, the Carpathian Basin and the Balkans to Macedonia andd Bulgaria (Csuzdi \& Zicsi 2003).

\section{Dendrobaena proandra Omodeo \& Rota, 1989}

Dendrobaena proandra Omodeo \& Rota, 1989: 193. Csuzdi et al. 2006: 12. Misırlığlu et al. 2008: 80. Misırlığlu 2009: 22.

Distribution. Turkey (Csuzdi et al. 2006).

\section{Dendrobaena ressli Zicsi, 1973}

Dendrobaena ressli Zicsi, 1973: 220. Csuzdi et al. 2006: 14. Misırlıoğlu et al. 2008: 80. Misırlığlu 2009: 22.

Distribution. Turkey (Csuzdi et al. 2006).

\section{Dendrobaena retrosella Szederjesi \& Csuzdi, 2012}

Dendrobaena retrosella Szederjesi \& Csuzdi, 2012a: 33. Trakić et al. 2016: 262. Szederjesi et al. 2017a: 66.

Distribution. Greece (Szederjesi et al. 2017a).

\section{Dendrobaena rhodopensis (Černosvitov, 1937)}

Eisenia rhodopensis Černosvitov, 1937a: 82.
Dendrobaena rhodopensis: Mršić 1991: 617. Karaman \& Stojanović 1995: 140. Šapkarev 1997: 105. Stojanović \& Karaman 2003: 56. Stojanović et al. 2012: 10.; 2013: 639. Valchovski 2012: 93. Stojanović \& Milutinović 2013: 153. Trakić et al. 2016: 263. Szederjesi et al. 2017a: 66.

Dendrobaena durmitorensis Mršić, 1988: 15. Mršić 1991: 617. Šapkarev 1993: 17.; 1997: 105. Stojanović \& Milutinović 2013: 152. Trakić et al. 2016: 260.

Distribution. Bulgaria, Greece, Montenegro, Serbia (Mršić 1991).

Remarks. D. rhodopensis was first described from Bulgaria. Later Mršić (1988) described a similar species from Durmitor Mts. Montenegro, thought to be differring from $D$. rhodopensis in the position of the spermathecae. According to Mršić (1988) the spermathecae of D. rhodopensis open in setal line $d$ while those of $D$. durmitorensis near to the mediodorsal line. However, in the original description Černosvitov (1937) clearly states that the spermathecae "münden... dicht neben der dorsalen Medianlinie". Consequently D. durmitorensis was regarded as a synonym of D. rhodopensis (Csuzdi 2012). Before synonymization, D. rhodopensis was recorded even from the Durmitor Mts. by Stojanović \& Karaman (2003).

\section{Dendrobaena rothschildae Csuzdi \& Pavlíček, 1998}

Dendrobaena rothschildae Csuzdi \& Pavlíček, 1998: 26. Pavlíček et al. 2003: 456.

Distribution. Israel (Pavlíček et al. 2003).

\section{Dendrobaena samarigera (Rosa, 1893)}

Allolobophora samarigera Rosa, 1893a: 5.

Dendrobaena samarigera: Pavlíček et al. 2003: 456. Csuzdi et al. 2006: 14. Misırlığlu et al. 2008: 80. Misırlığlu 2009: 22. Szederjesi et al. 2013b: 204.

Distribution. ?Turkey, Israel, Lebanon (Pavlíček et al. 2003). 


\section{Dendrobaena schmidti marinae Kvavadze, 1985}

Dendrobaena schmidti marinae Kvavadze, 1985: 129. Csuzdi et al. 2006: 14. Misırlığlu et al. 2008: 80. Misırlığlu 2009: 22.

Distribution. Georgia, Turkey (Kvavadze 1985, Csuzdi et al. 2006).

\section{Dendrobaena schmidti tellermanica Perel, 1966}

Dendrobaena schmidti tellermanica Perel, 1966: 163. Csuzdi et al. 2006: 14. Misırlığlu et al. 2008: 80. Misirlığlu 2009: 22.

Dendrobaena surbiensis: Misırlığlu 2009: 22.

Distribution. Georgia, Armenia, Azerbaijan, Dagestan, Turkey (Kvavadze 1985, Csuzdi et al. 2006).

\section{Dendrobaena semitica (Rosa, 1893)}

Allolobophora semitica Rosa, 1893a: 3.

Dendrobaena semitica: Pavlíček et al. 2003: 456. Csuzdi \& Pavlíček 2005a: 73. Csuzdi et al. 2006: 14.; 2007: 351. Pavlíček \& Csuzdi 2006a: 184.; 2006b: S114.; 2017: 597. Misırlığlu et al. 2008: 80. Misırlığlu 2009: 22. Szederjesi et al 2013a: 395.; 2013b: 204.; 2014a: 560.; 2016.

Distribution. Turkey (Hatay), Cyprus, Israel, Lebanon, Syria, Jordan (Pavlíček et al. 2003).

\section{Dendrobaena serbica Karaman, 1973}

Dendrobaena serbica Karaman, 1973: 178.; Mršić 1991: 603. Šapkarev 1993: 17.; 1997: 105. Trakić et al. 2016: 263. Stojanović et al. 2017a: 183. 2014)

Distribution. Serbia, Montenegro (Szederjesi

\section{Dendrobaena skipetarica Szederjesi \& Csuzdi, 2017}

Dendrobaena skipetarica Szederjesi \& Csuzdi, 2017 in: Szederjesi et al. 2017b.

Dendrobaena ganglbaeri (part.): Szederjesi \& Csuzdi 2012b: 265.

Distribution. Albania (Szederjesi \& Csuzdi 2012b).
Dendrobaena szalokii Szederjesi, Pavlíček, Coşkun \& Csuzdi 2014

Dendrobaena szalokii Szederjesi, Pavlíček, Coşkun \& Csuzdi, 2014: 561.

Distibution: Turkey (Szederjesi et al. 2014a).

Dendrobaena transjordanica Szederjesi, Pavlíček \& Csuzdi, 2013

Dendrobaena transjordanica Szederjesi, Pavlíček \& Csuzdi, 2013a: 395.

Distribution. Jordan (Szederjesi et al. 2013a).

\section{?Dendrobaena vejdovskyi (Černosvitov, 1935)}

Bimastus vejdovskyi Černosvitov, 1935: 66.

Dendrobaena vejdovskyi: Mršić 1991: 592. Milutinović et al. 2010: 630. Stojanović et al. 2017a: 183.

Distribution. Austria, Germany, Slovakia, Hungary (Csuzdi \& Zicsi 2003), ?Serbia.

Remarks. According to our present knowledge D. vejdovskyi is a typical Eastern-Alpine species (Csuzdi \& Zicsi 2003). Its occurrence in Serbia needs further corroboration.

\section{Dendrobaena veneta (Rosa, 1886)}

Allolobphora veneta Rosa, 1886: 674.

Dendrobaena veneta: Csuzdi \& Pavlíček 2002: 111. Pavlíček \& Csuzdi 2006a: 184.; 2017: 596. Dhora 2010: 82. Stojanović et al. 2012: 10.; 2013: 639. Stojanović \& Milutinović 2014: 308. Szederjesi et al. 2016.

Dendrobaena veneta veneta: Mršić 1991: 613. Šapkarev 1993: 17. Karaman \& Stojanović 1995: 141. Pavlíček et al. 2003: 456. Stojanović \& Karaman 2003b: 56. Csuzdi \& Pavlíček 2005a: 75. Csuzdi et al. 2006: 15.; 2007: 354. Pavlíček \& Csuzdi 2006b: S114.; 2008: 194. Misırlığlu 2009: 22. Szederjesi \& Csuzdi 2012b: 268. Hackenberger Kutuzović \& Hackenberger Kutuzović 2013: 11. Stojanović \& Milutinović 2013: 154. Szederjesi 2013a: 69.; 2013b: 79.; 2014: 50. Szederjesi et al. 2013a: 396.; 2013b: 205.; 2014a: 560.; 2017a: 66. Stojanović et al. 2017a: 183.

Distribution. Widely introduced peregrine species (Csuzdi \& Zicsi 2003). 


\section{Dendrobaena zicsi Karaman, 1973}

Dendrobaena zicsi Karaman, 1973: 178.

Dendrobaena zicsii: Mršić 1991: 590. Šapkarev 1993: 17.; 1997: 105. Trakić et al. 2016: 263.

Distribution. Serbia (Mršić 1991).

Genus Dendrodriloides Kvavadze, 2000

\section{Dendrodriloides grandis grandis (Michaelsen, 1907)}

Eisenia grandis Michaelsen, 1907: 87.

Eophila grandis grandis: Omodeo 1988: 77.

Eisenia grandis grandis: Csuzdi et al. 2006: 16. Misırlığlu et al. 2008: 80. Misırlığlu 2009: 22.

Dendrodriloides grandis grandis: Csuzdi 2012.

Distribution. Georgia, Armenia, Turkey (Kvavadze 1985, Csuzdi et al. 2006).

\section{Dendrodriloides hydrophilicus (Kvavadze, 1979)}

Eisenia grandis hydrophilica Kvavadze, 1979: 143.

Eisenia hydrophilica: Csuzdi et al. 2006: 18. Misırlığlu et al. 2008: 80. Misırlığlu 2009: 22.

Dendrodriloides hydrophilicus: Csuzdi 2012.

Distribution. Turkey, Georgia (Omodeo \& Rota 1989).

\section{Dendrodriloides polysegmenticus (Kvavadze, 1973)}

Eisenia perelae polysegmentica Kvavadze, 1973: 6. Eophila grandis polysegmentica: Omodeo 1988: 78.

Eisenia grandis polysegmentica: Csuzdi et al. 2006: 18. Misırlıoğlu et al. 2008: 80. Misırlıŏlu 2009: 22.

Dendrodriloides polysegmenticus: Csuzdi 2012.

Distribution. Turkey, Georgia (Omodeo \& Rota 1989).

\section{Genus Eisenia Malm, 1877}

\section{Eisenia ariadne (Michaelsen, 1928)}

Eophila ariadne Michaelsen, 1928: 289.
Dendrobaena ariadne: Šapkarev 1997: 105.

Eisenia ariadne: Trakić et al. 2016: 263. Szederjesi et al. 2017a: 67.

Distribution. Greece (Naxos) (Szederjesi et al. 2017a).

\section{Eisenia colchidica (Perel, 1967)}

Eiseniella colchidica Perel, 1967: 101. Csuzdi et al. 2006: 18. Misırlıoğlu 2009: 22.

Eisenia colchidica: Mršić 1991: 507. Szederjesi et al. 2017a: 67.

Distribution. Georgia, Turkey (Omodeo \& Rota 1991).

\section{Eisenia ebneri (Michaelsen, 1914)}

Helodrilus venetus var. ebneri Michaelsen, 1914: 8. Eisenia grandis ebneri: Mršić 1991: 509. Šapkarev 1997: 104.

Eisenia ebneri: Trakić et al. 2016: 263. Szederjesi et al. 2017a: 68 .

Distribution. Greece (Mršić 1991)

\section{Eisenia fetida (Savigny, 1826)}

Enterion fetidum Savigny, 1826: 182.

Eisenia foetida: Karaman \& Stojanović 1995: 140. Šapkarev 2001: 111. Stojanović \& Karaman 2003b: 57. Misırlıoğlu 2009: 22.

Eisenia fetida: Mršić 1991: 497. Pavlíček et al. 2003: 456. Csuzdi \& Pavlíček 2005a: 75.; 2005b: 91. Pavlíček \& Csuzdi 2008: 194.; 2017: 592. Stojanović et al. 2012: 11.; 2013: 640.; 2017a: 184. Szederjesi \& Csuzdi 2012b: 269. Valchovski 2012: 94.; 2014: 4. Hackenberger Kutuzović \& Hackenberger Kutuzović 2013: 13. Stojanović \& Milutinović 2013: 155.; 2014: 308. Szederjesi 2013a: 69.; 2014: 50. Szederjesi et al. 2014a: 565.; 2016; 2017a: 68. Valchovski \& Szederjesi 2016: 358 .

Eisenia andrei (Bouché, 1972): Hackenberger Kutuzović \& Hackenberger Kutuzović 2013: 12. Valchovski \& Szederjesi 2016: 357.

Distribution. Widely distributed peregrine species (Csuzdi \& Zicsi 2003).

\section{Eisenia kattoulasi Zicsi \& Michalis, 1981}

Eisenia kattoulasi Zicsi \& Michalis, 1981: 254. Mršić 1991: 508. Šapkarev 1997: 104. Csuzdi et 
al. 2006: 18. Misırlığlu 2009: 22. Szederjesi et al. 2017a: 68 .

Distribution. Greece, Turkey (Szederjesi et al. 2017a).

\section{Eisenia lucens (Waga, 1857)}

Lumbricus lucens Waga, 1857: 161.

Eisenia lucens: Mršić 1991: 500. Karaman \& Stojanović 1995: 140. Šapkarev 2001: 111. Stojanović \& Karaman 2003b: 57.; 2005a: 130. Stojanović et al. 2012: 11.; 2013: 640.; 2017a: 184. Valchovski 2012: 94.; 2014: 4. Hackenberger Kutuzović \& Hackenberger Kutuzović 2013: 13. Stojanović \& Milutinović 2013: 155.; 2014: 308. Szederjesi 2013a: 70.; 2013b: 80.; 2014: 50. Valchovski \& Szederjesi 2016: 358.

Distribution. From the Pyrenees through the Alps and the Carpathians to Macedonia and Bulgaria (Csuzdi \& Zicsi 2003).

\section{Eisenia muranyii Szederjesi \& Csuzdi, 2015}

Eisenia muranyii Szederjesi \& Csuzdi, 2015: 112. Trakić et al. 2016: 264.

Distribution. Albania (Szederjesi \& Csuzdi 2015).

\section{Eisenia oreophila Szederjesi \& Csuzdi, 2012}

Eisenia oreophila Szederjesi \& Csuzdi, 2012a: 36. Trakić et al. 2016: 264. Szederjesi et al. 2017a: 68.

Distribution. Greece (Szederjesi \& Csuzdi 2012a).

\section{Eisenia spelaea (Rosa, 1901)}

Allolobophora spelaea Rosa, 1901: 36.

Eisenia spelaea: Mršić 1991: 503. Hackenberger Kutuzović \& Hackenberger Kutuzović 2013: 13. Szederjesi 2013a: 70. Stojanović \& Milutinović 2014: 308.

Distribution. Italy, Austria, Hungary, Slovenia, Croatia, Bosnia-Herzegovina, Montenegro (Csuzdi \& Zicsi 2003, Szederjesi 2013a).

\section{Eisenia storkani Černosvitov, 1934}

Eisenia rosea storkani Černosvitov, 1934a: 71.

Eisenia grandis storkani: Mršić 1991: 510. Šapkarev 1997: 104. Valchovski 2012: 94.

Eisenia storkani: Stojanović et al. 2013: 640. Szederjesi 2013b: 80. Trakić et al. 2016: 264.

Distribution. Bulgaria (Valchovski 2012).

\section{Genus Eiseniella Michaelsen, 1900}

\section{Eiseniella neapolitana (Örley, 1885)}

Allurus neapolitanus Örley, 1885: 12.

Eiseniella tetraedra neapolitana: Mršić 1991: 524. Pavlíček et al. 2003: 456. Csuzdi \& Pavlíček 2005a: 76.

Eiseniella neapolitana: Csuzdi \& Pavlíček 2005b: 92. Csuzdi et al. 2006: 19.; 2007: 355. Pavlíček \& Csuzdi 2006a: 185.; 2006b: S114.; 2008: 194.; 2017: 594. Misırlıoglu et al. 2008: 80. Misirlığlu 2009: 22. Szederjesi et al. 2013a: 398.; 2013b: 206.; 2017a: 68.

Distribution. From France through Macedonia, Greece and Turkey to Cyprus, Jordan and Dagestan (Pavlíček et al. 2003).

\section{Eiseniella ochridana ochridana Černosvitov, 1931}

Eiseniella ochridana Černosvitov, 1931a: 97. (part.)

Eiseniella ochridana ochridana: Mršić 1991: 525 (for complete synonymy). Šapkarev 1997: 104. Trakić et al. 2016: 264.

Distribution. Macedonia (Mršić 1991).

\section{Eiseniella ochridana profunda Černosvitov, 1931}

Eiseniella ochridana f. profunda Černosvitov, 1931a: 97.

Eiseniella ochridana profunda: Mršić 1991: 527. Šapkarev 1997: 104. Trakić et al. 2016: 264.

Distribution. Macedonia (Mršić 1991).

\section{Eiseniella tetraedra (Savigny, 1826)}

Enterion tetraedrum Savigny, 1826: 184.

Eiseniella tetraedra: Csuzdi \& Pavlíček 2005b: 91. Csuzdi et al. 2006: 19.; 2007: 355. Szederjesi et 
al. 2014a: 566.; 2017a: 69. Stojanović \& Milutinović 2014: 308. Valchovski \& Szederjesi 2016: 358.

Eiseniella tetraedra tetraedra: Mršić 1991: 514. Šapkarev 2001: 112. Pavlíček et al. 2003: 457. Csuzdi \& Pavlíček 2005a: 76. Pavlíček \& Csuzdi 2006b: S114.; 2008: 194.; 2016: 8. Misirlığlu 2009: 23. Valchovski 2012: 95.; 2014: 5. Stojanović et al. 2012: 11.; 2013: 640.

Eiseniella tetraeda: Pavlíček \& Csuzdi 2006a: 185. Dhora 2010: 82. Szederjesi \& Csuzdi 2012b: 269. Hackenberger Kutuzović \& Hackenberger Kutuzović 2013: 14. Stojanović \& Milutinović 2013: 155. Szederjesi 2013b: 80. Szederjesi et al. 2013a: 398; 2013b: 206. Stojanović et al. 2017a: 184.

Eiseniella tetraedra pupa (Eisen, 1874): Mršić 1991: 520. Šapkarev 2001: 112. Valchovski 2012: 95.

Eiseniella tetraedra intermedia Černosvitov, 1934: Mršić 1991: 523.

Eiseniella peleensis Tzelepe, 1943: Šapkarev 1997: 104.

Distribution. Widely distributed peregrine species (Csuzdi \& Zicsi 2003).

\section{Genus Healyella Omodeo \& Rota, 1989}

\section{Healyella baloghi (Zicsi, 1981)}

Bimastos baloghi Zicsi, 1981a: 435.

Healyella baloghi: Csuzdi et al. 2006: 20. Misirlıoğlu et al. 2008: 80. Misırlığlu 2009: 22.

Distribution. Turkey (Csuzdi et al. 2006).

\section{Healyella boluana Omodeo \& Rota, 1989}

Healyella boluana Omodeo \& Rota, 1989: 176. Csuzdi et al. 2006: 20. Misırlığlu et al. 2008: 80. Misırlığlu 2009: 22.

Distribution. Turkey (Csuzdi et al. 2006).

\section{Healyella jordanis (Csuzdi \& Pavlíček, 1999)}

Bimastos jordanis Csuzdi \& Pavlíček, 1999: 471.; 2002: 109. Pavlíček et al. 2003: 456.

Healyella jordanis: Szederjesi et al. 2013b: 206.

Distribution. Israel (Pavlíček et al. 2003).

\section{Healyella mariae Omodeo \& Rota, 1989}

Healyella mariae Omodeo \& Rota, 1989: 175. Csuzdi et al. 2006: 20. Misırlığlu et al. 2008: 80. Misırlığlu 2009: 22.

Distribution. Turkey (Csuzdi et al. 2006).

\section{Healyella michaelseni Omodeo \& Rota, 1989}

Healyella michaelseni Omodeo \& Rota, 1989: 174. Csuzdi et al. 2006: 20. Misırlığlu et al. 2008: 80. Misırlıŏlu 2009: 22.

Distribution. Turkey (Csuzdi et al. 2006).

\section{Healyella naja Omodeo \& Rota, 1989}

Healyella naja Omodeo \& Rota, 1989: 176. Csuzdi et al. 2006: 20. Misirlığlu et al. 2008: 81. Misırlığlu 2009: 22.

Distribution. Turkey (Csuzdi et al. 2006).

\section{Healyella schweigeri (Zicsi, 1981)}

Bimastos schweigeri Zicsi, 1981a: 434. Healyella schweigeri: Csuzdi et al. 2006: 22. Misırlığlu et al. 2008: 81. Misırlığlu 2009: 22.

Distribution. Turkey (Csuzdi et al. 2006).

\section{Healyella syriaca (Rosa, 1893)}

Allolobophora syriaca Rosa, 1893b: 461.

Healyella syriaca: Csuzdi et al. 2006: 22.; 2007: 355. Misırlığlu et al. 2008: 81. Misırlığlu 2009: 22. Szederjesi et al. 2013a: 398.; 2013b: 206.; 2014a: 566.

Bimastos syriacus: Pavlíček et al. 2003: 456.

Distribution. Turkey, Syria, Lebanon, Israel, Jordan, Iran (Pavlíček et al. 2003, Szederjesi et al. 2013a).

\section{Healyella zapparolii Omodeo \& Rota, 1989}

Healyella zapparolii Omodeo \& Rota 1989: 177. Csuzdi et al. 2006: 22. Misırlığlu et al. 2008: 81. Misırlıŏlu 2009: 22.

Distribution. Turkey (Csuzdi et al. 2006). 


\section{Genus Helodrilus Hoffmeister, 1845}

\section{Helodrilus balcanicus balcanicus (Černosvitov, 1931)}

Eiseniella balcanica Černosvitov, 1931b: 321.

Helodrilus balcanicus balcanicus: Mršić 1991: 108. Šapkarev 1997: 103. Stojanović et al. 2013: 640. Trakić et al. 2016: 265. Szederjesi et al. 2017a: 70.

Distribution. Serbia, Montenegro, Macedonia, Greece (Mršić 1991).

\section{Helodrilus balcanicus plavensis (Karaman, 1972)}

Eiseniella balcanica plavensis Karaman, 1972c: 78. Helodrilus balcanicus plavensis: Mršić 1991: 110. Šapkarev 1997: 103. Stojanović et al. 2013: 640. Stojanović \& Milutinović 2013: 156. Trakić et al. 2016: 265.

Distribution. Montenegro, Serbia (Mršić 1991).

\section{Helodrilus cernosvitovianus (Zicsi, 1967)}

Allolobophora cernosvitoviana Zicsi, 1967: 248.

Helodrilus cernosvitovianus: Mršić 1991: 115. Stojanović et al. 2013: 640. Szederjesi et al. 2017a: 70.

Distribution. Hungary, Ukraine, Poland, Serbia, Greece (Csuzdi \& Zicsi 2003).

\section{Helodrilus dinaricus Mršić, 1991}

Helodrilus dinaricus Mršić, 1991: 108. Šapkarev 1997: 103. Trakić et al. 2016: 265.

Distribution. Slovenia (Mršić 1991).

\section{Helodrilus duhlinskae Zicsi \& Csuzdi, 1986}

Helodrilus duhlinskae Zicsi \& Csuzdi, 1986: 119. Mršić 1991: 121. Šapkarev 1997: 103. Stojanović et al. 2012: 11. Valchovski 2012: 95. Trakić et al. 2016: 265.

Distribution. Bulgaria (Valchovski 2012).

\section{Helodrilus italicus Zicsi, 1985}

Helodrilus italicus Zicsi, 1985b: 284. Mršić 1991: 123.

Helodrilus serbicus: Mršić 1991: 120. Šapkarev 1997: 103. Trakić et al. 2016: 266.

Distribution. Italy, Serbia (Mršić 1991).

\section{Helodrilus jadronensis Šapkarev, 1989}

Helodrilus jadronensis Šapkarev, 1989: 36.; 1997: 103. Mršić 1991: 108. Hackenberger Kutuzović \& Hackenberger Kutuzović 2013: 14. Trakić et al. 2016: 265.

Distribution. Croatia (Mršić 1991).

\section{Helodrilus kratochvili (Černosvitov, 1937)}

Eophila kratochvili Černosvitov, 1937b: 130.

Helodrilus kratochvili: Mršić 1991: 114. Šapkarev 1997: 103. Szederjesi et al. 2014c: 182. Trakić et al. 2016: 265.

Distribution. Bosnia-Herzegovina (Mršić 1991).

\section{Helodrilus patriarchalis (Rosa, 1893)}

Allolobophora patriarchalis Rosa, 1893a: 9.

Helodrilus patriarchalis: Mršić 1991: 119. Šapkarev 1997: 103. Pavlíček et al. 2003: 457. Csuzdi \& Pavlíček 2005a: 76.; 2005b: 92. Csuzdi et al. 2006: 22.; 2007: 356. Pavlíček \& Csuzdi 2006a: 185.; 2006b: S114.; 2017: 596. Misırlıoğlu et al. 2008: 81. Misırlıŏlu 2009: 23. Szederjesi et al. 2013a: 398.; 2013b: 207.; 2014a: 566.; 2014c: 184.; 2017a: 71.

Helodrilus colchicus Kvavadze, 2000: 82. (Csuzdi 2012, Szederjesi et al. 2014c).

Helodrilus zicsianus Kvavadze, 2000: 83. (Csuzdi 2012, Szederjesi et al. 2014c).

Distribution. Greece, Turkey, Cyprus, Azerbaijan, Georgia, Syria, Lebanon, Israel, Jordan, Iran (Szederjesi et al. 2017a).

\section{Helodrilus vagneri Mršić, 1991}

Helodrilus vagneri Mršić, 1991: 116. Šapkarev 
1997: 103. Trakić et al. 2016: 266. Szederjesi et al. 2017a: 71.

Distribution. Bosnia-Herzegovina, Greece (Szederjesi et al. 2017a).

\section{Genus Lumbricus Linnaeus, 1758}

\section{Lumbricus castaneus Savigny, 1826}

Enterion castaneum Savigny, 1826: 180.

Lumbricus castaneus: Mršić 1991: 466. Karaman \& Stojanović 2002: 224. Dhora 2010: 82. Stojanović \& Milutinović 2013: 156. Szederjesi 2013a: 71.

Distribution. Widely distributed peregrine species (Csuzdi \& Zicsi 2003).

\section{Lumbricus improvisus Zicsi, 1963}

Lumbricus improvisus Zicsi, 1963: 75. Mršić 1991: 470. Šapkarev 1997: 104.

Distribution. Romania, Serbia, Macedonia (Mršić 1991).

\section{Lumbricus meliboeus Rosa, 1884}

Lumbricus meliboeus Rosa, 1884: 21. Mršić 1991: 471. Stojanović et al. 2012: 11. Hackenberger Kutuzović \& Hackenberger Kutuzović 2013: 16. Milutinović et al. 2013: 64. Stojanović \& Milutinović 2013: 156.

Distribution. Italy, Austria, Slovenia, Croatia, Bosnia-Herzegovina, Montenegro, Serbia, Bulgaria (Hackenberger Kutuzović \& Hackenberger Kutuzović 2013, Milutinović et al. 2013).

\section{Lumbricus polyphemus (Fitzinger, 1833)}

Enterion polyphemus Fitzinger, 1833: 552.

Lumbricus polyphemus: Mršić 1991: 473. Karaman \& Stojanović 2002: 224. Stojanović \& Karaman 2005a: 128. Stojanović et al. 2012: 11.; 2013: 640.; 2017a: 185. Valchovski 2012: 96. Hackenberger Kutuzović \& Hackenberger Kutuzović 2013: 16. Szederjesi 2013a: 71. Stojanović \& Milutinović 2014: 308.
Distribution. From Italy through Hungary, Romania, Slovenia, Croatia and Serbia to Bulgaria (Csuzdi \& Zicsi 2003).

\section{Lumbricus rubellus Hoffmeister, 1843}

Lumbricus rubellus Hoffmeister, 1843: 187. Mršić 1991: 474. Karaman \& Stojanović 1995: 141. Šapkarev 2001: 112. Stojanović \& Karaman 2003b: 56.; 2005a: 130. Csuzdi et al. 2006: 23. Misırlığlu 2009: 23. Valchovski 2012: 96.; 2014: 5. Stojanović et al. 2012: 11.; 2013: 640.; 2017a:185. Hackenberger Kutuzović \& Hackenberger Kutuzović 2013: 16. Stojanović \& Milutinović 2013: 157.; 2014: 308. Szederjesi 2013a: 71.; 2014: 50. Szederjesi \& Csuzdi 2015: 113. Szederjesi et al. 2017a: 71.

Distribution. Widely distributed peregrine species (Csuzdi \& Zicsi 2003).

\section{Lumbricus terrestris Linnaeus, 1758}

Lumbricus terrestris Linnaeus, 1758: 647. Mršić 1991: 481. Stojanović \& Karaman 2003b: 56. Stojanović et al. 2012: 11.; 2013: 640. Valchovski 2012: 96; 2014: 5. Stojanović \& Milutinović 2013: 157.; 2014: 308. Szederjesi 2013b: 80.; 2014: 50. Valchovski \& Szederjesi 2016: 358 .

Distribution. Widely distributed peregrine species (Csuzdi \& Zicsi 2003).

\section{Genus Murchieona Gates, 1978}

\section{Murchieona minuscula (Rosa, 1905)}

Allolobophora minuscula Rosa, 1905: 38.

Murchieona minuscula: Mršić 1991: 535. Csuzdi \& Pavlíček 2002: 108. Pavlíček et al. 2003: 457. Csuzdi et al. 2006: 23.; 2007: 356. Pavlíček \& Csuzdi 2006b: S114.; 2017: 594. Misırlığlu et al. 2008: 81. Misirlığlu 2009: 23. Hackenberger Kutuzović \& Hackenberger Kutuzović 2013: 17. Szederjesi et al. 2013b: 207.; 2014a: 567.; 2017a: 71. Valchovski \& Misırlığlu 2017: 732 .

Distribution. Widely distributed in the Mediterranean (Pavlíček \& Csuzdi 2017). 


\section{Genus Octodriloides Zicsi, 1986}

\section{Octodriloides bolei Mršić, 1987}

Octodriloides bolei Mršić, 1987b: 88.; 1991: 449. Šapkarev 1997: 104.; Hackenberger Kutuzović \& Hackenberger Kutuzović 2013: 17.

Distribution. Slovenia, Croatia (Hackenberger Kutuzović \& Hackenberger Kutuzović 2013).

\section{Octodriloides bosniensis Mršić, 1991}

Octodriloides bosniensis Mršić, 1991: 455. Šapkarev 1997: 104. Trakić et al. 2016: 266.

Distribution. Bosnia-Herzegovina (Mršić 1991).

\section{Octodriloides dinaricus Mršić, 1991}

Octodriloides dinaricus Mršić, 1991: 445. Šapkarev 1997: 104.

Distribution. Slovenia (Mršić 1991).

\section{Octodriloides janetscheki (Zicsi, 1970)}

Octolasium (Octodrilus) janetscheki Zicsi, 1970: 171.

Octodriloides janetscheki: Mršić 1991: 450. Šapkarev 1997: 104. Hackenberger Kutuzović \& Hackenberger Kutuzović 2013: 17.

Distribution. Slovenia, Croatia (Hackenberger Kutuzović \& Hackenberger Kutuzović 2013).

\section{Octodriloides kamnensis (Baldasseroni, 1919)}

Octolasium complanatum f. kamnensis Baldasseroni, 1919: 1.

Octodriloides kamnensis: Mršić 1991: 458. (part.). Hackenberger Kutuzović \& Hackenberger Kutuzović 2013: 18.

Octodriloides camnensis: Šapkarev 1997: 104. (sic!)

Distribution. Slovenia, Croatia (Hackenberger Kutuzović \& Hackenberger Kutuzović 2013).

\section{Octodriloides karawankensis (Zicsi, 1969)}

Octolasium (Octodrilus) karawankensis Zicsi, 1969: 382.

100
Octodriloides kamnensis: Mršić 1991: 458 (part.).

Octodriloides karawankensis: Csuzdi \& Zicsi 2003: 221.

Distribution. Italy, Austria, Hungary, Slovenia and Croatia (Mršić 1991, Csuzdi \& Zicsi 2003).

Remarks. Oc. karawankensis is a typical Southern Alpine species. The only occurrence on the Balkan is from Mršić (1991: 461).

\section{Octodriloides kovacevici (Zicsi, 1970)}

Octolasium (Octodrilus) kovacevici Zicsi, 1970: 169.

Octodriloides kovacevici: Mršić 1991: 452. Šapkarev 1997: 104. Hackenberger Kutuzović \& Hackenberger Kutuzović 2013: 18. Szederjesi 2013a: 72.

Distribution. Slovenia, Croatia (Hackenberger Kutuzović \& Hackenberger Kutuzović 2013).

\section{Octodriloides marinceki Mršić, 1987}

Octodriloides marinceki Mršić, 1987b: 91.; 1991: 444. Šapkarev 1997: 104. Trakić et al. 2016: 266.

Distribution. Slovenia (Mršić 1991).

\section{Octodriloides poklonensis Mršić, 1991}

Octodriloides poklonensis Mršić, 1991: 443. Šapkarev 1997: 104. Hackenberger Kutuzović \& Hackenberger Kutuzović 2013: 18. Trakić et al. 2016: 266.

Distribution. Croatia (Hackenberger Kutuzović \& Hackenberger Kutuzović 2013).

\section{Genus Octodrilus Omodeo, 1956}

\section{Octodrilus albanicus Szederjesi \& Csuzdi, 2012}

Octodrilus albanicus Szederjesi \& Csuzdi, 2012b: 271. Trakić et al. 2016: 266.

Distribution. Albania (Szederjesi \& Csuzdi 2012b). 


\section{Octodrilus bretscheri (Zicsi, 1969)}

Octolasium bretscheri Zicsi, 1969: 72.

Octodrilus bretscheri: Mršić 1991: 369. Hackenberger Kutuzović \& Hackenberger Kutuzović 2013: 18. Stojanović \& Milutinović 2013: 157.

Distribution. Italy, Austria, Croatia, Macedonia, Serbia, Montenegro (Hackenberger Kutuzović \& Hackenberger Kutuzović 2013).

\section{Octodrilus complanatus (Dugès, 1828)}

Lumbricus complanatus Dugès, 1828: 289.

Octodrilus complanatus: Mršić 1991: 398. Šapkarev 2002: 305. Karaman \& Stojanović 2002: 224. Pavlíček et al. 2003: 457. Stojanović \& Karaman 2003b: 56.; 2005a: 130. Csuzdi \& Pavlíček 2005b: 92. Csuzdi et al. 2006: 24. Pavlíček \& Csuzdi 2006b: S114.; 2008: 194.; 2017: 594. Misırlığlu et al. 2008: 81. Misırlıŏlu 2009: 22. Dhora 2010: 82. Stojanović et al. 2012: 11.; 2017a: 185. Szederjesi \& Csuzdi 2012b: 270. Valchovski 2012: 96. Hackenberger Kutuzović \& Hackenberger Kutuzović 2013: 19. Stojanović \& Milutinović 2013: 158.; 2014: 308. Szederjesi 2013a: 72.; 2013b: 81. Szederjesi et al. 2014a: 567.; 2017a: 72.

Distribution. Widely distributed in the whole Mediterranean (Pavlíček \& Csuzdi 2016).

\section{Octodrilus croaticus (Rosa, 1895)}

Allolobophora lissaensis var. croatica Rosa, 1895: 5.

Octodrilus croaticus: Mršić 1991: 393. Dhora 2010: 82. Szederjesi \& Csuzdi 2012b: 279. Hackenberger Kutuzović \& Hackenberger Kutuzović 2013: 19. Szederjesi et al. 2017a: 72.

Distribution. Italy, Austria, Croatia, Albania, Greece (Hackenberger Kutuzović \& Hackenbeger Kutuzović 2013).

\section{Octodrilus kvarnerus Mršić, 1987}

Octodrilus kvarnerus Mršić, 1987b: 39.; 1991: 385. Šapkarev 1997: 104. Hackenberger Kutuzović \& Hackenberger Kutuzović 2013: 20. Trakić et al. 2016: 267.
Distribution. Croatia (Hackenberger Kutuzović \& Hackenberger Kutuzović 2013).

\section{Octodrilus lissaensis (Michaelsen, 1891)}

Allolobophora lissaensis Michaelsen, 1891: 18.

Octodrilus lissaensis: Mršić 1991: 381. Hackeberger Kutuzović \& Hackenberger Kutuzović 2013: 20. Stojanović \& Milutinović 2013: 158. Szederjesi 2013a: 72.; 2014: 51.

Octolasium lissaense: Karaman \& Stojanović 1995: 142.

Octolasion lissaense: Stojanović \& Karaman 2003b: 57.

Distribution. From Italy to Romania and through Slovenia, Croatia to Bosnia-Herzegovina and Montenegro (Hackenberger Kutuzović \& Hackenberger Kutuzović 2013).

\section{Octodrilus mimus (Rosa, 1889)}

Allolobophora mima Rosa, 1889: 1.

Octodrilus mimus: Mršić 1991: 411. Hackenberger Kutuzović \& Hackenberger Kutuzović 2013: 20. Trakić et al. 2016: 267.

Octodrilus mima: Šapkarev 1997: 104.

Distribution. Italy, Slovenia, Croatia (Hackenberger Kutuzović \& Hackenberger Kutuzović 2013).

\section{Octodrilus oesophagus Mršić, 1991}

Octodrilus oesophagus Mršić, 1991: 388. Šapkarev 1997: 104. Trakić et al. 2016: 267.

Distribution. Slovenia (Mršić 1991).

\section{Octodrilus pseudolissaensis Mršić, 1991}

Octodrilus pseudolissaensis Mršić, 1991: 426. Šapkarev 1997: 104. Hackenberger Kutuzović \& Hackenberger Kutuzović 2013: 21.

Distribution. Croatia (Hackenberger Kutuzović \& Hackenberger Kutuzović 2013).

\section{Octodrilus pseudotranspadanus (Zicsi, 1971)}

Octolasium (Octodrilus) pseudotranspadanum Zicsi, 1971: 227. 
Octodrilus pseudotranspadanus: Mršić 1991: 377. Hackenberger Kutuzović \& Hackenberger Kutuzović 2013: 21.

Distribution. Hungary, Croatia, Bosnia-Herzegovina (Csuzdi \& Zicsi 2003).

\section{Octodrilus rucneri (Plisko \& Zicsi, 1970)}

Octolasium (Octodrilus) rucneri Plisko \& Zicsi, 1970: 454.

Octodrilus rucneri: Mršić 1991: 418. Šapkarev 1997: 104. Hackenberger Kutuzović \& Hackenberger Kutuzović 2013: 21. Trakić et al. 2016: 267.

Distribution. Italy, Croatia (Hackenberger Kutuzović \& Hackenberger Kutuzović 2013).

\section{Octodrilus savinensis Mršić, 1987}

Octodrilus savinensis Mršić, 1987a: 37.; 1991: 420.

Distribution. Slovenia (Mršić 1991).

\section{Octodrilus slovenicus (Karaman, 1972)}

Octolasium mima var. slovenica Karaman, 1972a: 102.

Octodrilus slovenicus: Mršić 1991: 428. Hackenberger Kutuzović \& Hackenberger Kutuzović 2013: 22.

Octodrilus slovenucus: Šapkarev 1997: 104.

Distribution. Slovenia, Croatia (Hackenberger Kutuzović \& Hackenberger Kutuzović 2013).

\section{Octodrilus tergestinus (Michaelsen, 1910)}

Octolasium mima var. tergestina Michaelsen, 1910a: 73.

Octodrilus tergestinus: Mršić 1991: 417. Šapkarev 1997: 104. Hackenberger Kutuzović \& Hackenberger Kutuzović 2013: 22. Trakić et al. 2016: 267.

Distribution. Italy, Slovenia, Croatia (Hackenberger Kutuzović \& Hackenberger Kutuzović 2013).

\section{Octodrilus transpadanus (Rosa, 1884)}

Allolobophora transpadana Rosa, 1884: 45.

Octodrilus transpadanus: Mršić 1991: 371. Csuzdi \& Pavlíček 2005a: 76. Csuzdi et al. 2006: 24.; 2007: 357. Pavlíček \& Csuzdi 2006a: 185. Misırlıoğlu et al. 2008: 81. Misırlıŏlu 2009: 23. Dhora 2010: 82. Stojanović et al. 2012: 11.; 2013: 640.; 2017a: 185. Szederjesi \& Csuzdi 2012b: 270.; 2015: 114. Valchovski 2012: 97.; 2014: 6. Hackenberger Kutuzović \& Hackenberger Kutuzović 2013: 22. Stojanović \& Milutinović 2013: 59.; 2014: 308. Szederjesi 2013a: 72.; 2013b: 81. Szederjesi et al. 2013a: 398.; 2014a: 567.; 2017a: 73.

Distribution. From Switzerland, Italy to Turkey (Csuzdi \& Zicsi 2003).

\section{Octodrilus transpadanoides Zicsi, 1981}

Octodrilus transpadanoides Zicsi, 1981b: 161.; Mršić 1991: 376.

Distribution. Slovenia (Mršić 1991).

\section{Octodrilus velebiticus Mršić, 1991}

Octodrilus velebiticus Mršić, 1991: 404. Šapkarev 1997: 104. Hackenberger Kutuzović \& Hackenberger Kutuzović 2013: 23. Trakić et al. 2016: 267.

Distribution. Croatia (Hackenberger Kutuzović \& Hackenberger Kutuzović 2013).

\section{Genus Octolasion Örley, 1885}

\section{Octolasion cyaneum (Savigny, 1826)}

Enterion cyaneum Savigny, 1826: 181.

Octolasion cyaneum: Mršić 1991: 345. Šapkarev 2002: 304. Pavlíček \& Csuzdi 2006b: S114.; 2017: 592. Hackenberger Kutuzović \& Hackenberger Kutuzović 2013: 23. Stojanović \& Milutinović 2014: 308.

Distribution. Widely distributed peregrine species (Csuzdi \& Zicsi 2003). 


\section{Octolasion lacteum (Örley, 1881)}

Lumbricus terrestris var. lacteus Örley, 1881: 584.

Octolasium lacteum: Karaman \& Stojanović 1995: 141.

Octolasion lacteum: Šapkarev 2001: 111. Stojanović \& Karaman 2003b: 56.; 2005a: 130. Csuzdi et al. 2006: 24. Dhora 2010: 82. Stojanović et al. 2012: 11.; 2013: 640.; 2017a: 185. Valchovski 2012: 98.; 2014: 6. Hackenberger Kutuzović \& Hackenberger Kutuzović 2013: 23. Stojanović \& Milutinović 2013: 159.; 2014: 308. Szederjesi 2013a: 72.; 2013b: 81.; 2014: 51. Szederjesi \& Csuzdi 2015: 114. Valchovski \& Szederjesi 2016: 358. Szederjesi et al. 2017a: 73.

Octolasion tyrtaeum: Mršić 1991: 347. Misırlığlu 2009: 23.

Distribution. Widely distributed peregrine species (Csuzdi \& Zicsi 2003).

\section{Genus Perelia Easton, 1983}

\section{Perelia aharonii (Stephenson, 1922)}

Helodrilus (Allolobophora) aharonii Stephenson, 1922: 136.

Allolobophora (s.l.) aharonii: Pavlíček et al. 2003: 456.

Perelia aharonii: Csuzdi \& Pavlíček 2005b: 79.

Distribution. Israel (Pavlíček et al. 2003).

\section{Perelia biokovica (Mršić, 1986)}

Allolobophora biokovica Mršić, 1986: 71;

Alpodinaridella (Dinaridella) biokovica: Mršić 1991: 240. Šapkarev 1997: 104. Hackenberger Kutuzović \& Hackenberger Kutuzović 2013: 24. Trakić et al. 2016: 267.

Distribution. Croatia (Hackenberger Kutuzović \& Hackenberger Kutuzović 2013).

\section{Perelia galileana Csuzdi \& Pavlíček, 2005}

Perelia galileana Csuzdi \& Pavlíček, 2005a: 81. Csuzdi et al. 2007: 357. Misırlığlu 2008: 81; 2009: 23. Szederjesi et al. 2013b: 207.

Distribution. Israel, Turkey (Hatay) (Csuzdi et al. 2007).
Perelia hatayica Csuzdi, Pavlíček \& Mısırlıoğlu, 2007

Perelia hatayica Csuzdi, Pavlíček \& Misırlıoğlu, 2007: 357. Misırlığlu et al. 2008: 81. M1sırlığlu 2009: 23. Szederjesi et al. 2014a: 567.

Distribution. Turkey (Hatay) (Csuzdi et al. 2007).

\section{Perelia makrisi Szederjesi, Pavlíček \& Csuzdi, 2016}

Perelia makrisi Szederjesi, Pavlíček \& Csuzdi 2016: 159.

Allolobophora nematogena: Pavlíček \& Csuzdi 2006: S114. (part.)

Perelia nematogena: Pavlíček \& Csuzdi 2016: 11. (part.)

Distribution. Cyprus (Szederjesi et al. 2016).

\section{Perelia nematogena (Rosa, 1903)}

Allolobophora nematogena Rosa, 1903: 11. Šapkarev 2002: 297. Pavlíček \& Csuzdi 2006b: S114.

Microeophila nematogena (part.): Mršić 1991: 221. Šapkarev 1997: 104.

Perelia nematogena: Stojanović et al. 2013: 640. Hackenberger Kutuzović \& Hackenberger Kutuzović 2013: 24. Stojanović \& Milutinović 2013: 159. Pavlíček \& Csuzdi 2017: 595.

Alpodinaridella (Alpodinaridella) lozniciana Mršić, 1987: Mršić 1991: 238. Šapkarev 1997: 104. syn. nov.

Perelia lozniciana: Trakić et al. 2016: 268.

Distribution. Italy, Hungary, Slovenia, Croatia, Bosnia-Herzegovina, Montenegro, Macedonia, Serbia (Csuzdi \& Zicsi 2003).

Remarks. Investigation of a large Pe. nematogena material housed in the Hungarian Natural History Museum collected in Italy (HNHM/ 6801, $6805,6841,6863,12805,12826,12830,12811)$, Hungary $(4122,4132,4135,4136,5403,9120)$, Croatia (6494, 6495), Slovenia (6743), Serbia (7642, 12799) and Bosnia-Herzegovina (6673) revealed that the position of the clitellum and the tubercles show a greater variance: (cl.) $1 / 224,25$, $1 / 225,26,1 / 226,27-33,1 / 234,34,1 / 235$ and (tb.) 
$1 / 228,29,1 / 229,30-32,1 / 233,33,1 / 234$. The clitellum and tubercles of Alpodinaridella lozniciana described by Mršić (1987) (cl: 25 - 1/2 34 and tb: $28-1 / 232$ ) largely overlap with these value ranges; its other characteristics are completely similar with Pe. nematogena as well, including its typical hook-shaped nepridial bladders bearing a terminal ampulla. Therefore, Alpodinaridella lozniciana is regarded here as a synonym of Perelia nematogena.

On the other hand, in the characters of Microeophila nematogena (Rosa, 1903) Mršić (1991: 221) does not mention the existence of an ectal ampulla on the nephridial bladders, therefore we can not decide obviously whether the specimens identified by Mršić as Microeophila nematogena belong to Pe. nematogena or represent a different species.

\section{Perelia phoebea (Cognetti, 1913)}

Helodrilus (Allolobophora) phoebeus Cognetti, 1913: 2.

Aporrectodea (Aporrectodea) jassyensis phoebea: Mršić 1991: 320.

Allolobophora nematogena Rosa, 1903: Pavlíček \& Csuzdi 2006: S114. (part.).

Perelia nematogena: Pavlíček \& Csuzdi 2017: 595. (part.).

Perelia phoebea: Szederjesi et al. 2016: 160.; 2017a: 73. Trakić et al. 2016: 268.

Distribution. Greece (Rhodes), Cyprus (Szederjesi et al. 2016, 2017a).

\section{Perelia shamsi Csuzdi \& Pavlíček, 2005}

Perelia shamsi Csuzdi \& Pavlíček, 2005a: 84. Szederjesi et al. 2013b: 207.

Distribution. Israel (Csuzdi \& Pavlíček 2005a).

\section{Genus Proctodrilus Zicsi, 1985}

\section{Proctodrilus antipai (Michaelsen, 1891)}

Allolobophora antipae Michaelsen, 1891: 16.

Proctodrilus antipai antipai: Valchovski 2012: 98.
Proctodrilus antipai: Mršić 1991: 131. Stojanović et al. 2012: 11.; 2017a: 186. Hackenberger Kutuzović \& Hackenberger Kutuzović 2013: 24. Stojanović \& Milutinović 2014: 308. Szederjesi et al. 2017a: 74.

Distribution. From France to the Black Sea (Csuzdi \& Zicsi 2003).

\section{Proctodrilus opisthoductus Zicsi, 1985}

Proctodrilus opisthoductus Zicsi, 1985b: 147. Mršić 1991: 130. Zicsi \& Michalis 1993: 303. Šapkarev 1997: 103. Szederjesi 2013a: 72. Stojanović \& Milutinović 2014: 308. Szederjesi et al. 2017a: 74.

Distribution. Austria, Slovenia, Serbia, Greece (Csuzdi \& Zicsi 2003).

\section{Proctodrilus tuberculatus (Černosvitov, 1935)}

Eophila antipae var. tuberculata Černosvitov, 1935b: 58

Proctodrilus tuberculatus: Mršić 1991: 134. Csuzdi et al. 2006: 24. Pavlíček \& Csuzdi 2006b: S114.; 2008: 194.; 2017: 598. Misırlığlu et al. 2008: 81. Misırlıoğlu 2009: 23. Stojanović et al. 2013: 640. Hackenberger Kutuzović \& Hackenberger Kutuzović 2013: 25. Stojanović \& Milutinović 2014: 308. Szederjesi et al. 2016.; 2017a: 74.

Proctodrilus tuberculata: Stojanović et al. 2012: 11.

Proctodrilus antipai tuberculatus: Valchovski 2012: 98.

Distribution. From France and Germany to the Western Caucasus (Csuzdi \& Zicsi 2003).

Genus Spermophorodrilus Bouché, 1975

\section{Spermophorodrilus antiquus (Černosvitov, 1938)}

Allolobophora antiqua Černosvitov, 1938b: 198.

Spermophorodrilus antiquus: Dhora 2010: 82. Stojanović et al. 2012: 11. Szederjesi et al. 2017a: 74.

Spermophorodrilus antiquus antiquus: Mršić 1991: 532. Šapkarev 1997: 104. Trakić et al. 2016: 268. 
Spermophorodrilus antiquus bouchei: Mršić 1991: 533. Šapkarev 1997: 104.

Spermophorodrilus antiquus michalisi: Mršić 1991: 532. Šapkarev 1997: 104.

Distribution. Albania, Greece, Bulgaria (Szederjesi et al. 2017a).

Spermophorodrilus simsoni Omodeo \& Rota, 1989

Spermophorodrilus simsoni Omodeo \& Rota, 1989: 172. Csuzdi et al. 2006: 25. Misirlığlu et al. 2008: 81. Misırlığlu 2009: 22.

Distribution. Turkey (Csuzdi et al. 2006).

Spermophorodrilus vignai Omodeo \& Rota, 1989

Spermophorodrilus vignai Omodeo \& Rota, 1989: 171.; Csuzdi et al. 2006: 25. Misırlığlu et al. 2008: 81. Misırlığlu 2009: 22.

Distribution. Turkey (Csuzdi et al. 2006).

Genus Trapezonscolex Qiu \& Bouché, 1998 stat. nov.

Eophila (Trapezonscolex) Qiu \& Bouché, 1998: 195.

Trapezonscolex cavazzutii cavazzutii Omodeo, 1988 comb. nov.

Eophila cavazzutii Omodeo, 1988: 75.

Eophila cavazzutii cavazzutii: Csuzdi et al. 2006: 19. Misırlığlu et al. 2008: 80. Misırlıoğlu 2009: 23.

Distribution. Turkey (Csuzdi et al. 2006).

Trapezonscolex cavazzutii pascuorum Omodeo, 1988 comb. nov.

Eophila cavazzutii pascuorum Omodeo, 1988: 76. Csuzdi et al. 2006: 19. Misırlığlu et al. 2008: 80. Misırlığlu 2009: 23.

Distribution. Turkey (Csuzdi et al. 2006).
Remarks. Qiu \& Bouché (1998) created the subgenus Trapezonscolex for Eo. cavazzutii cavazzutii and Eo. c. pascuorum on the basis of some characters differing from the other Eophila species $e g$. the lack of dorsal pores, 2 or 3 pairs of vesicles, the presence of testic sacs.

The original description (Omodeo 1988) does not mention the nephridial bladders, but after investigating Omodeo's specimens, Bouché stated that the orientation of the nephridial bladders of Eophila is 'reclinate'. Unfortunately, it did not turn out from the text whether he studied the Turkish cavazzutii specimens or Eo. tellinii (Rosa, 1988). Examination of one preadult cavazzutii specimen in the collection of the Hungarian Natural History Museum (HNHM/7918) collected in Derel1, Vil. Giresun, Turkey, revealed that its nephridial bladders are 'reclinate' which clearly separates this species from Eo. tellinii and Eo. crodabepis Paoletti, 2016 bearing 'proclinate' bladders (Paoletti et al. 2016). Therfore, on the basis of the above mentioned characteristics, separation of the subgenus Trapezonscolex from Eophila and raising it to genus level is proposed.

\section{Species incertae sedis}

\section{Allolobophora eurytanica (Tzelepe, 1943)}

Eophila eurytanica Tzelepe, 1943: 1.

Allolobophora eurytanica: Trakić et al. 2016: 255. Szederjesi et al. 2017a: 76.

Distribution. Greece (Zicsi \& Michalis 1981).

Remarks. A. eurytanica was described with cl: on $1 / 230-36$ and tb: on 35,36 . It has a large male pore on 15 and two pairs of spermathecae in $9 / 10-10 / 11 d$. The species has never been reported after the original description. The position of the clitellum and the large male pores suggest its similarity to A. chlorotica. Normally A. chlorotica possesses three pairs of tubercles on $31,33,35$ however, in anomalous specimens these tubercles can vary in number and position therefore, we 
cannot exclude that eutrytanica is just an anomalous A. chlorotica specimen.

\section{Dendrobaena sasensis Šapkarev, 1993}

Dendrobaena sasensis Šapkarev, 1993: 22.; 1997: 105. Mršić 1991: 603. Trakić et al. 2016: 263.

Distribution. Macedonia (Mršić 1991).

Remarks. The tubercles of this species are on segments 31-32, in all other characters it resembles $D$. attemsi. As the position of the tubercles can vary within $30-1 / \mathrm{n} 32,1 / 232,32$ in case of the latter species, we can't obviously exclude that $D$. sasensis is in fact $D$. attemsi especially if the nephridiopores - which are not mentioned in the original description - alternate regularly.

\section{Family Acanthodrilidae Claus, 1880}

\section{Genus Dichogaster Beddard, 1888}

\section{Dichogaster bolaui (Michaelsen, 1891)}

Benhamia bolavi Michaelsen, 1891: 9.

Dichogaster bolaui: Pavlíček et al. 2003: 457.

Distribution. Widely distributed peregrine species (Csuzdi 2010).

\section{Genus Microscolex Rosa, 1887}

\section{Microscolex dubius (Fletcher, 1887)}

Eudrilus dubius Fletcher, 1887: 378.

Microscolex dubius: Pavlíček et al. 2003: 457. Szederjesi et al. 2014a: 568; 2017a: 75.

Distribution. Widely distributed peregrine species (Blakemore 2008).

\section{Microscolex phosphoreus Dugès, 1837}

Lumbricus phosphoreus Dugès, 1837: 17.

Microscolex phosphoreus: Pavlíček et al. 2003: 457. Szederjesi et al. 2017a: 75.

Distribution. Widely distributed peregrine species (Blakemore 2008).
Family Criodrilidae Vejdovsky, 1884

Genus Criodrilus Hoffmeister, 1845

\section{Criodrilus lacuum Hoffmeister, 1845}

Criodrilus lacuum Hoffmeister, 1845: 41. Pavlíček et al. 2003: 457. Csuzdi et al. 2007: 358. Szederjesi et al. 2014a: 568.; 2017a: 74.

Distribution. Widely distributed peregrine species (Blakemore 2008).

\section{Family Megascolecidae Rosa, 1891}

Genus Amynthas Kinberg, 1867

\section{Amynthas corticis (Kinberg, 1867)}

Perichaeta corticis Kinberg, 1867: 102.

Amynthas corticis: Szederjesi et al. 2017a: 75.

Distribution. Widely distributed peregrine species (Blakemore 2008).

\section{Amynthas gracilis (Kinberg, 1867)}

Nitocris gracilis Kinberg, 1867: 102.

Amynthas gracilis: Szederjesi et al. 2017a: 75.

Distribution. Peregrine species found from tropical to warm-temperate localities (Blakemore 2008).

Genus Metaphire Sims \& Easton, 1972

\section{Metaphire californica (Kinberg, 1867)}

Pheretima californica Kinberg, 1867: 102.

Metaphire californica: Pavlíček et al. 2003: 457.

Distribution. Widely distributed peregrine species (Blakemore 2008).

\section{Genus Pontodrilus Perrier, 1874}

\section{Pontodrilus litoralis (Grube, 1855)}

Lumbricus litoralis Grube, 1855: 127.

Pontodrilus litoralis: Szederjesi et al. 2017a: 76. 
Distribution. Widely distributed peregrine species (Blakemore 2008).

\section{Family Ocnerodrilidae Beddard, 1891}

Genus Eukerria Michaelsen, 1935

\section{Eukerria saltensis (Beddard, 1895)}

Kerria saltensis Beddard, 1895: 225.

Eukerria saltensis: Szederjesi et al. 2017a: 76.

Distribution. Widely distributed peregrine species (Blakemore 2008).

\section{Genus Ocnerodrilus Eisen, 1878}

\section{Ocnerodrilus occidentalis Eisen, 1878}

Ocnerodrilus occidentalis Eisen, 1878: 10. Pavlíček et al. 2003: 457. Szederjesi et al. 2017a: 76.

Distribution. Widely distributed pantropical species (Blakemore 2008).

\section{SUMMARY}

The first combined checklist of the earthworms of the Balkans, Anatolia, the Levant and Cyprus contains 226 species and subspecies, of which 216 belong to the family Lumbricidae, the dominant family in the Holarctic. The acanthodrilids take part with three, the megascolecids with four, the ocnerodrilids with two and the criodrilids with one species.

Altogether 27 species, including all non-lumbricids, are widely distributed peregrines (11.9\%). However it's worth to emphasize that some peregrine lumbricid species could also be members of the autochtonous fauna but nowadays it would be difficult to prove this.

Out of the 166 Balkanic species 90 occur solely on the Peninsula (54.2\%). Anatolia has 26 endemic species out of the 70 taxa present $(37.1 \%)$, while Levant has 14 out of the 42 (33.3\%). With 21 species present, Cyprus has only one endemic earthworm, Perelia makrisi Szederjesi, Pavlíček \& Csuzdi, 2016.

The family Lumbridicae is represented with 19 genera, of which Dendrobaena is the most speciose with 67 taxa. This clearly confirms the statement of Omodeo \& Rota (1989) that this genus has two of its three main distribution centres (Caucasus-Transcaucasus-Anatolia and the Balkan Peninsula-Carpathian Basin) on the area studied.

Studying the literature resulted in finding several synonym names in the region, e.g. Allolobophora kosowensis montenegrina (=A. kosowensis kosowensis), Dendrobaena alpina mavrovensis $(=D$. alpina alpina $), D$. olympica peristerica $(=D$. olympica olympica) and Alpodinaridella lozniciana $(=$ Perelia nematogena $)$.

On the basis of the orientation of nephridial bladders ('reclinate'), lack of dorsal pores and the number of seminal vesicles ( 2 or 3 pairs) we proposed raising the subgenus Trapezonscolex to genus level for the former Turkish Eophila species Eo. cavazzutii cavazzutii and Eo. cavazzutii pascuorum.

Acknowledgement. I would like to thank Csaba Csuzdi (Eszterházy Károly University, Eger, Hungary) for his useful comments on the manuscript. Many thanks to Mirjana Stojanović (University of Kragujevac, Kragujevac, Serbia) for providing morphological data on A. kosowensis kosowensis and A. kosowensis montenegrina specimens of her collection and the anonymous reviewers whose comments greatly helped to improve the manuscript.

\section{REFERENCES}

BALDASSERONI, V. (1919): Helodrilus (Eophila) chinagliae n. sp. ed altri lombrichi del museo civico di Genova. Annali del Museo Civico de Storia Naturale di Genova, 8: 1-9.

BEDDARD, F.E. (1895): A monograph of the order of Oligochaeta. Clarendon Press, Oxford, $721 \mathrm{pp}$.

BLAKEMORE, R.J. (2004): A provsional list of valid names of Lumbricoidea (Oligochaeta) after Easton, 1983. In. Moreno, A.G. \& Borges, S. (Eds.) Advances in Earthworm Taxonomy, Editorial Complutense, Madrid, p. 75-120. 
BLAKEMORE, R.J. (2006): A list of valid, invalid and synonymous names of Criodriloidea and Lumbricoidea [Annelida: Oligochaeta: Criodrilidae (inc. Biwadrilidae), Sparganophilidae, Ailoscolecidae (inc. Komarekionidae), Hormogastridae, Lumbricidae, Lutodrilidae]. In. A Series of Searchable Texts on Earthworm Biodiversity, Ecology and Systematics from Various Regions of the World 2nd Edition (2006). KANEKO, N. \& ITO, M.T. (Eds.) COE Soil Ecology Research Group, Yokohama National University, Japan. CD-ROM.

BLAKEMORE, R.J. (2008): Cosmopolitan earthworms an Eco-Taxonomic Guide to the Species (3rd Edition). VermEcology, Yokohama, Japan, 757 pp.

ČERnOsvitov, L. (1930): Zur Kenntnis der Oligochätenfauna des Balkans I. Zoologischer Anzeiger, 86: 319-333.

ČERnOSVITOV, L. (1931a): Zur Kenntnis der Oligochätenfauna des Balkans. II. Die wasserbewohnenden Lumbriciden aus dem Ochridasee. Zoologischer Anzeiger, 95: 96-103.

ČERNOSVITOV, L. (1931b): Zur Kenntnis der Oligochätenfauna des Balkans. III. Oligochäten aus Montenegro und Südserbien. Zoologischer Anzeiger, 95: 312-327.

ČERnOSVITOV, L. (1934a): Die Lumbriciden Bulgariens. Mitteilungen aus den Königlich Naturwissenschaftlichen Instituten in Sofia, 7: 71-78.

ČERnOSVITOV, L. (1934b): Sur les Oligochètes terricoles de Crète. Sbornik Zoologického Oddeleni Narodniho Musea v Praze, 1(4): 17-20.

ČERnOSVITOV, L. (1935a): Zur Kenntnis der Oligochätenfauna des Balkans IV. Höhlen-Oligochäeten aus Jugoslawien. Zoologischer Anzeiger, 111(5/6): 265-266.

ČERnOSVITOV, L. (1935b): Monographie des tschechoslovakischen Lumbriciden. Archiv pro Prirodovedecky Vyzkum Cech, 19: 1-86.

ČERNOSVITOV, L. (1937a): Die Oligochaetenfauna Bugariens. Mitteilungen aus den Königlich Naturwissenschaftlichen Instituten in Sofia, 10: 62-92.

ČERNOSVITOV, L. (1937b): Notes sur les Oligochaetes cavernicoles. Mémoires de la Société Zoologique Tchécoslovaque de Prague, 5: 125-133.

ČERnosvitov, L. (1938a): The Oligochaeta. In. Washbourn, R. \& Jones, R. F. (Eds.) Report of the Percy Sladen Expedition to Lake Huleh; a contribution to the study of fresh waters of
Palestine. Annals and Magazine of Natural History, 11(2): 535-550. doi: 10.1080/00222933808526881

ČERNOSVITOV, L. (1938b): Zur Kenntnis der Oligochätenfauna des Balkans VI. Oligochäten aus Grichenland. Zoologischer Anzeiger, 123(7/9): 192-200.

ČERnosvitov, L. (1940): On some Oligochaeta from Palestine. Annals and Magazine of Natural History, 11(6): 438-447. doi: 10.1080/03745481.1940.9723700

Chinaglia, L. (1913): Escursioni zoologiche del Dr. E. Festa. Lumbricidae. Bollettino dei Musei di zoologia ed anatomia comparata della R. Università di Torino, 28(667): 1-6.

CognetTI, L. (1906): Nuovi dati sui Lumbricidi dell'Europa orientale. Bollettino dei Musei di zoologia ed anatomia comparata della R. Università di Torino, 21(257): 1-18.

CognetTi, L. (1913): Escursioni zoologiche del Dr. E. Festa nell'Isola di Rodi V. Oligocheti. Bollettino dei Musei di zoologia ed anatomia comparata della R. Università di Torino, 28(74): 1-6.

CSUZDI, Cs. (2010). A monograph of the Paleotroplical Benhamiinae earthworms (Annelida: Oligochaeta, Benhamiinae). Hungarian Natural History Museum, Budapest, 348 pp.

CSUZDI, Cs. (2012): Earthworm species, a searchable database. Opuscula Zoologica, Budapest, 43(1): 97-99.

CSUZDI, Cs. \& PAVLÍČEK, T. (1999): Earthworms from Israel. I. Genera Dendrobaena Eisen, 1874 and Bimastos Moore, 1893 (Oligochaeta: Lumbricidae). Israel Journal of Zoology, 45: 467-486.

CSUZDI, Cs. \& PAVLÍČEK, T. (2002): Murchieona minuscula (Rosa, 1906), a newly recorded earthworm from Israel, and distribution of the genera Dendrobaena and Bimastos in Israel (Oligochaeta, Lumbricidae). Zoology in the Middle East, 25: 105-114. doi: 10.1080/09397140.2002.10637911

CsuzDI, Cs. \& PAVLÍČEK, T. (2005a): Earthworms from Israel. II. Remarks on the genus Perelia Easton, 1983 with description of a new genus and two new species. Acta Zoologica Academiae Scientiarum Hungaricae, 51(2): 75-96.

Csuzdi, Cs. \& PAVlíčEK, T. (2005b): Earthworms of Jordan. Zoology in the Middle East, 34: 71-77 doi: 10.1080/09397140.2005.10638085

CsUZDI, Cs \& POP, V.V. (2007): Redescription of Allolobophora dugesi getica Pop, 1947 and its 
allocation to the genus Cernosvitovia Omodeo, 1956 (Oligochaeta Lumbricidae). European Journal of Soil Biology, 43: S19-S23. doi: 10.1016/j.ejsobi.2007.08.005

CsuzDI, Cs. \& POP, V.V. (2008): Taxonomic and biogeographic analysis of the Allolobophora sturanyi species group (Oligochaeta, Lumbricidae). Opuscula Zoologica Budapest, 37: 23-28.

CsuZDI, Cs. \& ZICSI, A. (2003): Earthworms of Hungary (Annelida: Oligochaeta; Lumbricidae). Hungarian Natural History Museum, Budapest, 271 pp.

Csuzdi, Cs., PAvlíčEK, T. \& Nevo, E. (1998): A new earthworm species, Dendrobaena rothschildae sp. n. from Israel, and comments on the distribution of Dendrobaena species in the Levant (Oligochaeta: Lumbricidae). Opuscula Zoologica Budapest, 31: 25-32.

CsuZdi, Cs., Zicsi, A. \& MisirlioĞLU, M. (2006): An annotated checklist of the earthworm fauna of Turkey (Oligochaeta: Lumbricidae). Zootaxa, 1175: $1-29$.

Csuzdi, Cs., PAVlíČEK, T. \& MisiRlioĞLU, M. (2007): Earthworms (Oligochaeta: Lumbricidae, Criodrilidae and Acanthodrilidae) of Hatay Province, Turkey, with description of three new lumbricids. Acta Zoologica Academiae Scientiarum Hungaricae, 53(4): 347-361.

Csuzdi, Cs., Pop, V.V. \& PoP, A.A. (2011): The earthworm fauna of the Carpathian Basin with new records and description of three new species (Oligochaeta: Lumbricidae). Zoologischer Anzeiger, 250: 2-18. doi: 10.1016/j.jcz.2010.10.001

Csuzdi, Cs., Chang, C-H., PAvlíčeK, T., SzederJesi, T., EsOPI, D. \& SzLÁVECZ, K. (2017): Molecular phylogeny and systematics of native North American lumbricid earthworms (Clitellata: Megadrili). Plos One, 12(8): e0181504. doi: 10.1371/journal.pone.0181504

DHORA, D. (2010): Register of the species of the fauna of Albania. Camaj-Pipa Publisher, Skhodra, 208 pp.

DUGÈs, A. (1828): Recherche sur la circulation, la respiration, et la reproduction des Annélides sétigéres abranches. Annales des Sciences Naturelles Paris, 15: 284-336.

DUGÈs, A. (1837): Nouvelles observations sur la zoologie et l'anatomie des Annélides sétigéres abranches. Annales des Sciences Naturelles Paris ser. 2 Zoologie, 8: 15-35.
EASTON, E.G. (1983): A guide to the valid names of Lumbricidae (Oligochaeta). In. SATCHELL, J.E. (Ed.) Earthworm Ecology, from Darwin to vermiculture. Chapman \& Hall, London, p. 467487.

EISEN, G. (1874): New Englands och Canadas Lumbricider. Öfversigt af Kongliga VetenskapsAkademiens Förhandligar, 31(2): 41-49.

EISEN, G. (1878): On the Anatomy of Ocnerodrilus. Nova Acta Regiae Societatis Scientiarum Upsaliensis, 10(4): 1-12.

FITZINGER, L. (1833): Beobachtungen über die Lumbrici. Isis, 4: 549-553.

FLETCHER, J.J. (1987): Notes on Australian earthworms. Part III. Proceedings of the Linnean Society of New south Wales, 2(2): 375-402.

GRIFFITHS, H.I., KRYŠTUFEK, B. \& REED, M. (2004): Balkan Biodiversity. Patterns and processes in the European Hotspot. Kluwer, Boston, London. doi: 10.1007/978-1-4020-2854-0

GRUBE, E. (1855): Beschreibungen neuer oder wenig bekannter Anneliden. Archiv für Naturgeschichte, 27: $81-136$.

HACKENBERGER KutuZOVIĆ, D. \& HACKENBERGER KuTUZOVIĆ, B. (2013): Checklist of the earthworm fauna of Croatia (Oligochaeta: Lumbricidae). Zootaxa, 3710(1): 1-30. doi: 10.11646/zootaxa.3710.1.1

HOFFMEISTER, W. (1843): Beitrag zur Kenntnis deutcher Landanneliden. Archiv für Naturgeschichte, 91: 183-198.

HOFFMEISTER, W. (1845): Übersicht aller bis jetzt bekannten Arten aus der Familie der Regenwürmer. Friedrich Vieweg \& Sohn, Braunschweig, pp. 43.

KARAMAN, S. (1968): Über eine neue Regenwürm-Art aus Serbien, Allolobophora kosowensis n. sp., Zoologischer Anzeiger, 181(1/2): 50-53.

KARAMAN, S. (1969): Ein Beitrag zur Kenntnis der Lumbricidenfauna Mazedoniens. Zoologischer Anzeiger, 182: 75-83.

KARAMAN, S. (1972a): Beitrag zur Kenntnis der Oligochaetenfauna Jugoslawiens. Bioloski Vestnik, 20: 95-105.

KARAMAN, S. (1972b): Beitrag zur Kenntnis der Lumbricidenfauna von Griechenland. Fragmenta Balcanica Skopje, 9(11): 109-115. 
KARAMAN, S. (1972c): Beitrag zur Kenntnis der Art Eiseniella balcanica Černosvitov, 1931 (Oligochaeta, Lumbricidae). Fragmenta Balcanica Skopje, 9(7): 77-80.

KARAMAN, S. (1973): Drugi prilog poznavanju kišnih glista Srbije. Zbornik Radova, 1: 177-182.

KARAMAN, S. (1983): The third contribution to the knowledge of the earthworms of Serbia. Zbornik radova, Drugi simpozium o fauni SR Srbije, Beograd, 51-53.

KARAMAN, S.\& STOJANOviĆ, M. (1995): Contribution to the knowledge on the earthworms (Oligochaeta: Lumbricidae) in Montenegro. Archives of Biological Sciences, 47(3-4): 139-143.

KARAMAN, S. \& StOJAnOviĆ, M. (1996): New earthworm (Oligochaeta: Lumbricidae) records from Serbia (Yugoslavia). Bios (Macedonia, Greece), 4: 7-13.

KARAMAN, S. \& Stojanović, M. (2002): Treći prilog poznavanju kišnih glista (Oligochaeta, Lumbricidae) južne i jugoistočne Srbije. 7th Symposium on Flora of Southeastern Serbia and Neighbouring Regions, Proceedings, p. 223-225.

KINBERG, J.G.H. (1867): Annulata nova. Öfversigt af Kongliga Vetenskaps-Akademiens Förhandlingar, Stockholm, 23: 97-103, 356-357.

KVAVADZE, E. (1973): Studies on the Georgean Earthworms (Lumbricidae). Materiali $k$ Faune Gruzii, Mecniereba, Tblilisi, 3: 5-16. [in Russian]

KVAVADZE, E. (1979): Earthworms from moutain brown soils of Georgia. In. GELOVANIL L.V.T (Ed.) The Invertebrates of Brown and Mountain Balck Soils of Georgia. Mecniereba, Tblilisi, p. 143-157 [in Russian]

KVAVADZE, E. (1985): The earthworms (Lumbricidae) of the Caucasus. Metsniereba, Tbilisi, 238 pp. [in Russian]

Kvavadze, E. (2000): New Data on Earthworm (Oligochaeta: Lumbricidae) Systematics. Proceedings of the Institute of Zoology, 20: 77-84. [in Russian]

LEVINSEN, G.M.R. (1884): Systematisk-geografisk oversigtover de nordiske Annulata, Gephyrea, Chaetognathiog Balanoglossi. Videnskabelige Meddelelserfra den naturhistoriske Forening $i$ Kjöbenhavn, 45: 92-384.
LinNAEUS, C. (1758): Systema Naturae per Regna tria Naturae, secundum Classes, Ordines, Genera, Species, cum Characteribus, Differentiis, Synonymis, Locis. 10th edition, volume 1. Laurentii Salvii, Holmiae, 824 pp.

MAHUnKa, S., MuránYI, D. \& Kontschán, J. (2013): The role of the Balkan Peninsula in the origin and genesis of the soil fauna of the Carpathian Basin: history, aims and results. Opuscula Zoologica Budapest, 44(suppl. 1.): 5-10.

MiCHAELSEN, W. (1890): Die Lumbriciden Norddeutschlands. Jahrbuch der Hamburgischen Wissenschaftlichen Anstalten, 7: 1-19.

MiCHAELSEN, W. (1891): Oligochaeten des Naturhistorischen Museums in Hamburg IV. Jahrbuch der Hamburgischen Wisseenschaftlichen Anstalten, 8: $1-42$.

MiCHAELSEN, W. (1901): Oligochaeten der Zoologischen Museen zu St. Petersburg und Kiev. Bulletin de l'Académie Impériale des Sciences de St. Pétersbourg, 15: 136-215.

MiCHAELSEN, W. (1902): Neue Oligochaeten und neue Fundorte altbekannter. Mitteilungen aus dem Naturhistorischen Museum in Hamburg, 19: 3-53.

MICHAELSEN, W. (1903): Die geographische Verbreitung der Oligochaeten. Friedländer \& Sohn, Berlin, $186 \mathrm{pp}$.

MiCHAELSEN, W. (1907): Die Lumbriciden des Kaukasischen Museums in Tiflis. Mitteilungen des Kaukasischen Museums, 3: 81-93.

MICHAELSEN, W. (1910a): Oligochäten von verschiedenen Gebieten. Mitteilungen aus dem Naturhistorischen Museum in Hamburg, 27: 47-169.

MichaELSEN, W. (1910b): Zur Kenntniss der Lumbriciden und ihrer Verbreitung. Annuaire du Musée Zoologique de l'Académie Impériale des Sciences de St.-Pétersbourg, 15: 1-74.

MiCHAELSEN, W. (1914): Ein neuer Regenwurm aus Griechenland. Verhandlungen der ZoologischBotanischen Gesellschaft in Wien, 8: 8-9.

MichaELSEN, W. (1928): Beiträge zur Kenntnis der Fauna Griechenlands, namentlich der ägäischen Inseln. Sitzungsberichte der Kaiserlichen Akademie der Wissenschaften in Wien, 137: 289-290.

Michalis, K. (1993): Contribution to the study of the Oligochaete fauna of Cyprus. Acta zoologica cracoviensia, 36: 23-28. 
MichaLIS, K. (1995): Oligochaeten - Funde aus Thessalien (Griechenland) nebst Beschreibung der Art Octodrilus peleensis sp. nov. Bios (Macedonia, Greece), 3: 15-20.

Milutinović, T., Avramović, S., Pešić, S., Blesić, B., Stojanović, M. \& Bogdanović, A.M. (2010): Contribution to the knowledge of pedofauna in Šumadija (central part of Serbia). Biotechnology \& Biotechnological Equipment, 24(2SE.): 628-635.

Milutinović, T., Tsekova, R., Milanović, J. \& STOJANOVIĆ, M. (2013): Distribution, biogeographical significance and status of Lumbricus meliboeus Rosa, 1884 (Oligochaeta, Lumbricidae) at the European scale: first findings in Serbia and in Bulgaria. North-Western Journal of Zoology, 9(1): 63-69.

Milutinović, T., Milanović, J. \& Stojanović, M. (2015): Threat status and distribution of the endemic species Allolobophora kosowensis kosowensis Karaman, 1968 (Oligochaeta: Lumbricidae) in the Balkans. Journal of Natural History, 49: 471-481. doi: 10.1080/00222933.2013.791946

MisirlioĞLU, M. (2008): Some Earthworm Records from Anatolia (Oligochaeta, Lumbricidae). Turkish Journal of Zoology, 32: 469-471.

MisiRlioĞLU, M. (2009): Current checklist of terrestrial Turkish earthworms (Oligochaeta). Megadrilogica, 13(3): 21-24.

MisiRlioĞLU, M. (2010): Distribution of endemic earthworm species in Turkey (Oligochaeta: Lumbricidae). Zoology in the Middle East, 51(2): 83-87. doi: 10.1080/09397140.2012.10648990

MISIRLIOĞLU, M. (2012): Distribution of earthworms belonging to families Acanthodrilidae, Criodrilidae, and Megascolecidae in Turkey. Zoology in the Middle East, 58(4): 103-106. doi: 10.1080/09397140.2012.10648990

MisiRlioĞLU, M., PAVlíčEK, T. \& CSUZDI, Cs. (2008): Earthworm biodiversity in Turkey: An overview. In. PAVLÍČEK, T. \& CARDET, P. (eds.): Advances in Earthworm Taxonomy III. The Environment Service of the Ministry of Agriculture, Natural Resources and Environment of Cyprus, pp. 139161.

MisirlioğLu, M., Stojanović, M. \& Tsekova, R. (2017): Species richness of the earthworm fauna (Clitellata: Acanthodrilidae, Lumbricidae) of the Marmara region in Turkey: zoogeographical overview. North-Western Journal of Zoology, e172801, in press.

MRŠIĆ, N. (1982): Allolobophora altimontana sp. n. (Oligochaeta, Lumbricidae), a new species in Slovenia. Biološki Vestnik, 30(2): 57-62.

MrŠIĆ, N. (1986): Allolobophora biokovica sp. n. (Oligochaeta: Lumbricidae) and earthworm associations of the Biokovo (Coratia). Biološki Vestnik, 34(1): 69-80.

MRŠIĆ, N. (1987a): Description of a New Genus and Five Species of Earthworms (Oligochaeta: Lumbricidae), Scopolia, Ljubljana, 13: 1-11.

MRŠIĆ, N. (1987b): Description of three new species of earthworms of the genus Octodriloides Zicsi, 1986 (Oligochaeta: Lumbricidae). Biološki Vestnik, 35(2): 87-94.

MRŠIĆ, N. (1988): Description of five new species of earthworms of the genus Dendrobaena Eisen, 1874 (Lumbicidae). Biološki Vestnik, Ljubljana, 36(1): 13-24.

MRŠIĆ, N. (1990): Description of a new subgenus, three new species and taxonomic problems of the genus Allolobophora sensu Mršić and Šapkarev 1988 (Lumbricidae, Oligochaeta). Biološki Vestnik, 38(1): 49-68.

MRŠIĆ, N. (1991): Monograph on earthworms (Lumbricidae) of the Balkans I-II. Slovenska Akademija Znanosti in Umetnosti, Zazred za Naravoslovne Vede Opera 31 Ljubljana, 757 pp.

MRŠIĆ, N. (1992): Revision of the genera Cernosvitovia Omodeo, 1956, Italobalkaniona Mršić and Šapkarev, 1988 and Allolobophora Eisen, 1874 from the Balkans. Miscellanea Zoologica Barcelona, 16: 21-28.

MRŠIĆ, N. \& ŠAPKAREV, J., (1987): Survey of earthworms (Lumbricidae) of Serbia in a restricted sense and description of a new taxa. Biološki Vestnik, 35(2): 67-86.

OMODEO, P. (1952): Materiali zoologici raccolti dal Dr. Marcuzzi sulle Alpi Dolomitiche. Archivio Zoologico Italiano, 37: 29-59.

OMODEO, P. (1956): Oligocheti dell'Indochina e del Mediterraneo Orientale. Memorie del Museo Civico di Storia Naturale di Verona, 5: 321-336.

OMODEO, P. (1988): The genus Eophila (Lumbricidae, Oligochaeta). Bolletino di Zoologia, 55: 73-88 doi: 10.1080/11250008809386603 
OModeO, P. \& RotA, E. (1989): Earthworms of Turkey. Bollettino di Zoologia, 56: 167-199. doi: 10.1080/11250008909355639

OMOdEO, P. \& RotA, E. (1991): Earthworms of Turkey. II. Bollettino di Zoologia, 58: 171-181. doi: 10.1080/11250009109355749

ÖRLEY, L. (1881): A magyarországi Oligochaeták faunája. I. Terricolae. Mathematikai és Természettudományi Közlemények, 16: 562-611.

ÖRLEY, L. (1885): A palaearktikus övben élő Terrikoláknak revíziója és elterjedése. Értekezések a Természettudományok Köréböl, 15: 1-34.

Paoletti, M.G., BlaKemore, R.J., Csuzdi, Cs., DoRIGO, L., LEANDRO DREON, A., GAVINELLI, F., LAZZArini, F., Manno, M., Moretto, E., Porco, D., Ruzzier, E., Toniello, V., SQuartini, A., ConCHERI, G., ZANARDO, M. \& ALBA-TERCEDOR, J. (2016): Barcoding of Eophila crodabepis sp. nov. (Annelida, Oligochaeta, Lumbricidae), a Large Stripy Earthworm from Alpine Foothills of Northeastern Italy Similar to Eophila tellinii (Rosa, 1888). Plos One, 11(3): e0151799 doi: 10.1371/journal.pone.0151799

PAVLÍČEK, T. \& CSUZDI, CS. (2006a): Earthworm fauna of Jordan - A review. In. PoP, V.V. \& PoP, A.A. (Eds.) Advances in earthworm taxonomy II. (Annelida: Oligochaeta), University Press, Cluj, p. 183-188.

PAVlíčEK, T., \& CsuZDI, Cs. (2006b): Species richness and zoogeographic affinities of earthworms of Cyprus. European Journal of Soil Biology, 42: S111-S116. doi: 10.1016/j.ejsobi.2006.09.001

PAVlíČEK, T. \& CsuZdI, Cs. (2008): Does the autochthonous earthworm fauna emigrate from the Levant to Cyprus? In. PAVLÍČEK, T. \& CARDET, P. (eds.): Advances in Earthworm Taxonomy III (Annelida: Oligochaeta), Nicosia: En Tipis Voula Kokkinou Ltd., p. 189-200.

PAVlíČEK, T. \& CSUZDI, CS. (2017): Chapter 21: Clitellata: Oligochaeta. In. SPARROW, D.J., \& JOHN, E. (Eds.): An Introduction to the Wildlife of Cyprus, p. 586-599.

PAVlíčEK, T., CsuZdi, Cs. \& Nevo, E. (2003): Species richness and zoogeographic affinities of earthworms in the Levant. Pedobiologia, 47:452-457. doi: 10.1078/0031-4056-00212

PAVlíčEK, T., Csuzdi, Cs. \& Nevo, E. (2007): Biodiversity of earthworms in the Levant. Israel
Journal of Ecology and Evolution, 52(3-4): 385592. doi: 10.1560/IJEE_52_3-4_461

PEREL, T.S. (1966): Earthworms in the soil of forests of the N. W. Caucasus. In. RAYES, P.M. (Ed.) Influences of animals on the productivity of forest biogeocoenoses. Nauka Moscow, p. 146-165. [in Russian]

PEREL, T.S. (1967): Die Regenwürmer der Reliktwälder des westlichen Transkaukasien und des Talysch-Gebietes. Pedobiologia, 7: 93-120. [in Russian]

PlisKO, J.D. \& ZICSI, A. (1970): Octolasium (Octodrilus) rucneri n. sp., ein neuer Regenwurm aus Jugoslawien. Acta Zoologica Academiae Scientiarum Hungaricae, 16: 453-456.

POP, V. (1938): Neue Lumbriciden aus Rumänien. Buletinul Societății de Ştiințe din Cluj, 9: 134-152.

POP V. (1947): Allolobophora dugesi (Rosa) var. getica, une nouvelle variété de Lombricidé de la Roumanie. Comptes Rendus Biologies, Cluj, pp. 8-9.

POP, V. (1948): Allolobophora mehadiensis Rosa var. boscaiui, une nouvelle variété de Lumbricide et ses affinités. Buletinul Societății de Ştiințe din Cluj, 10: 104-109.

PoP, V. (1949): Lumbricidele din România. Analele Academiei Republicii Populare Române Secțiunea de Ştiințe Geologice, Geografice şi Biologie, 1(9): 383-505.

POP, V.V., POP, A.A. \& CsUZDI, Cs. (2012): An annotated checklist of the Romanian earthworm fauna (Oligochaeta, Lumbricidae). Zoology in the Middle East, 58(S4): 59-70. doi: $10.1080 / 09397140.2012 .10648985$

QIU, J-P. \& BouchÉ, M.B. (1998): Révision des taxons supraspécifiques de Lumbricoidea. Documents pédozoologiques \& intégrologiques, 3(6): 179-216.

REYNOLDS, J.W. \& CoOK, D.G. (1976): Nomenclatura Ologochaetologica. L'Université du NouveauBrunswick, Fredericton, 217 pp.

RosA, D. (1884): Lumbricidi del Piemonte. Torino, pp. 54.

RosA, D. (1886): Note sui lombrici del Veneto. Atti del Reale Istituto Veneto di Scienze, 4: 673-687.

RosA, D. (1889): Note sui Lombrichi iberici. Bollettino dei Musei di Zoologia ed Anatomia comparata della R. Universitŕ di Torino, 4(63): 1-5. 
RosA, D. (1892): Descrizione dell' Allolobophora smaragdina nuova specie di Lumbricide. Bollettino dei Musei di zoologia ed anatomia comparata della R. Università di Torino, 7(130): 1-2.

RosA, D. (1893a): Viaggio del Dr. E. Festa in Palestina, nel Libano e regioni vicine. - II. Lumbricidi. Bollettino dei Musei di Zoologia ed Anatomia comparata della $R$. Università di Torino, 8 (160): $1-14$.

RosA, D. (1893b): Revisione dei Lumbricidi. Memoires de l'Academie Royale des Sciences, Torino, 43: 399-476.

RosA, D. (1894): Allolobophora Ganglbaueri ed A. Oliveirae nuove specie di Lumbricidi europei. Bollettino dei Musei di Zoologia ed Anatomia comparata della $R$. Universitá di Torino, 9(170): 1-3.

RosA, D. (1895): Nuovi lombrichi dell' Europa orientale. Bolletino dei Musei di Zoologia ed Anatomia comparata della $R$. Università di Torino, 10(21): $1-8$.

RosA, D. (1897): Nuovi lombrichi dell'Europa orientale. (Seconda serie). Bollettino dei Musei di Zoologia ed Anatomia comparata della R. Universitá di Torino, 12(269): 1-5.

RosA, D. (1901): Un Lombrico cavernicolo (Allolobophora spelaea n. sp.). Atti della Società Naturalisti e Matematici di Modena, 4: 36-39.

RosA, D. (1903): L’Allolobophora (Eophila) nematogena n. sp. ed i suoi speciali linfociti. Atti della Societŕ dei Naturalisti e Matematici di Modena, 5: $11-13$.

RosA, D. (1905): Terricolen. In. Ergebnisse einer Naturwissenschaftlichen Reise zum Erdschias Dag. Annalen des Kaiserlich-Königlichen Naturhistorischen Hofmuseums Wien, 20: 104-106.

ŠAPKAREV, J. (1971): Neue Regenwürmer (Oligochaeta: Lumbricidae) aus Mazedonien. Fragmenta Balcanica Musei Macedonici Scientiarum Naturalium, 8(18): 149-164.

ŠAPKAREV, J. (1972a): Beitrage zur Kenntnis der Lumbricidenfauna Jugoslawiens. Archiv Bioloških Nauka, 24(1-2): 73-86.

ŠAPKAREV, J. (1972b): Beiträge zur Kenntnis der Lumbricidenfauna Griechenlands. Macedonian Academy of Sciences and Arts, 4: 31-36.

ŠAPKAREV, J. (1973): The Fauna of Earthworms of Macedonia. 4. The earthworms (Oligochaeta:
Lumbricidae) of the valley of Strumica. Godišen zbornik PMF, 25: 41-49.

ŠAPKAREV, J. (1975a): Neuere Angaben zur Kenntnis der Regenwürmer (Oigochaeta: Lumbricidae) aus Montenegro, Jugoslawien. Godisen Zbornik, Prirodno Matematicki fakultet, Univerzitet vo Skopje, 27-28: 27-38.

ŠAPKAREV, J. (1975b): Eine Neue Regenwurm-Art aus Serbien, Jugoslawien (Allolobophora paratuleskovi n. sp.), Godisen Zbornik, Prirodno Matematicki fakultet, Univerzitet vo Skopje, 27-28: 55-58.

ŠAPKAREV, J. (1975c): Contribution to the knowledge the earthworms (Lumbricidae) and leeches (Hirudinea) of Kosovo, Yugoslavia. Annuaire de la Faculté des Sciences de l'Université de Skopje, 2728: 39-54.

ŠAPKAREV, J. (1977a): Eophila pyrenaicoides sp. n., ein neuer regenwurm (Oligochaeta: Lumbricidae) aus Mazedonien. Fragmenta Balcanica Musei Macedonici Scientiarum Naturalium, 10(9): 75-78.

ŠAPKAREV, J. (1977b): The fauna of earthworms of Macedonia 7. The earthworms (Oligochaeta: Lumbricidae) of Ohrid-Struga valley. Annuaire de la Faculté des Sciences de l'Université de Skopje, 30: $28-45$.

ŠAPKAREV, J. (1989): Description of new species of earthworms (Oligochaeta: Lumbricidae) from Yugoslavia. Macedonian Academy of Sciences and Arts Contributions, 7(1-2): 33-46.

ŠAPKAREV, J. (1993): The species of the genera Dendrodrilus Omodeo, 1956, Dendrobaena Eisen, 1874 and Fitzingeria Zicsi, 1973 (Oligochaeta: Lumbricidae) of the lumbricid fauna in the territory of the former Yugoslavia. Fragmenta Balcanica Skopje, 15: 15-31.

ŠAPKAREV, J. (1997): High degree of endemicity of the lumbricid fauna in the Balkans. The University Thought - Publication in Natural Sciences (Pristina, Serbia), 4(2): 103-108.

ŠAPKAREV, J. (2001): Contribution to the knowledge of the fauna of Annelida from Pelister National Park, the Republic of Macedonia. In. BoŠKOVA, T. (Ed.) 75 years of Macedonian Museum of Natural History, Skopje, p. 107-124.

S̆APKAREV, J. (2002): Nove kišne gliste Srbije. Zbornik Radova SANU, 2002: 296-318.

SAVIGnY, J.C. (1826): In. CuvIER, G.: Analyse des Travaux de l'Academie royale des Sciences, pen- 
dant l'année 1821, partie physique. Mémoires de l'Académie des Sciences de l'Institut de France Paris, 5: 176-184.

STEPHENSON, J. (1922): On some earthworms from India and Palestine belonging to the British $\mathrm{Mu}-$ seum. Annals and Magazine of Natural Histrory, (9)9: 129-136. doi: 10.1080/00222932208632645

Stojanović, M. \& Karaman, S. (2003): Second contribution to the knowledge of earthworms (Lumbricidae) in Montenegro. Archives of Biological Sciences Belgrade, 55(1-2): 55-58.

STOJAnOvić, M. \& KARAMAN, S. (2005a): Further contribution to the knowledge of the earthworms of Šumadija (Serbia). Archives of Biological Sciences Belgrade, 57(2): 127-132.

Stojanović, M. \& KARAMAN, S. (2005b): Distribution of two species of the earthworm fauna of Sumadija (Serbia) in the Balkans and neighbouring territories. Archives of Biological Sciences Belgrade, 57(2): 123-136.

StOJANOVIĆ, M. \& KARAMAN, S. (2007): Distribution of endemic species from the earthworm genus Serbiona (Oligochaeta, Lumbricidae) in Serbia. Archives of Biological Sciences Belgrade, 59(2): 23-24.

Stojanović, M. \& Milutinović, T. (2013): Checklist of earthworms (Oligochaeta: Lumbricidae) of Montenegro: Diversity and biogeographical review. Zootaxa, 3710(2): 147-164. doi: 10.11646/zootaxa.3710.2

Stojanović, M. \& Milutinović, T. (2014): The earthworms (Oligochaeta: Lumbricidae) of the Pannonian region of Serbia, Vojvodina Province: Zoogeography and Diversity. North-Western Journal of Zoology, 10(2): 305-303.

Stojanović, M., Milutinové, T. \& Karaman, S. (2008): Earthworm (Lumbricidae) diversity in the Central Balkans: An evaluation of their conservation status. European Journal of Soil Biology, 44: 54-67. doi: 10.1016/j.ejsobi.2007.09.005

Stojanović, M., Tsekova, R. \& Milutinović, T. (2012): Earthworms (Oligochaeta: Lumbricidae) of Bulgaria: Diversity and Biogeographical Review. Acta Zoologica Bulgarica, Suppl. 4.: 7-15.

Stojanović, M., Tsekova, R., Pešić, S., Milanović, J. \& MilutinOviĆ, T. (2013): Diversity and a biogeographical review of the earthworms (Oligochaeta: Lumbricidae) of the Balkan Mountains
(Stara Planina Mountains) in Serbia and Bulgaria. Turkish Journal of Zoology, 37: 635-642. doi: 10.3906/zoo-1301-33

Stojanović, M., Trakić, T. \& SeKulić, J. (2017a): Earthworms of Kragujevac Basin - a review. Kragujevac Journal of Science, 39: 177-192.

Stojanović, M., SeKulić, J. \& Trakić, T. (2017b): Distribution and threat status of the endemic earthworm Allolobophora dofleini (Oligochaeta, Lumbricida) on the Balkan Peninsula NorthWestern Journal of Zoology, 13(1): 136-143.

SZEDERJESI, T. (2013a): New earthworm records from the former Yugoslav countries (Oligochaeta, Lumbricidae). Opuscula Zoologica Budapest, 44(1): 6176.

SZEDERJESI, T. (2013b): New earthworm records from Bulgaria (Oligochaeta, Lumbricidae). Opuscula Zoologica Budapest, 44(1): 77-83.

SZEDERJESI, T. (2014): Allolobophora ruzsai sp. n., a new earthworm species and new records from Montenegro (Oligochaeta: Lumbricidae). NorthWestern Journal of Zoology, 10(1): 48-52.

SZEDERJESI, T. (2015): New earthworm records from various parts of Greece (Oligochaeta: Lumbricidae, Acanthodrilidae, Megascolecidae, Ocnerodrilidae). Opuscula Zoologica Budapest, 46(2): 143-152. doi: 10.18348/opzool.2015.2.143

SzEDERJESI, T. (2017): Earthworms of Crete (Oligochaeta: Lumbricidae, Acanthodrilidae): new records, remarks and biogeographical review. NorthWestern Journal of Zoology, 13(1): 128-135.

SZEDERJESI, T. \& CSUZDI, Cs. (2012a): New and little known earthworm species from Greece (Oligochaeta: Lumbricidae, Acanthodrilidae). Zootaxa, 3304: 25-42.

SZEDERJESI, T. \& CsUZDI, Cs. (2012b): New earthworm species and records from Albania (Oligochaeta, Lumbricidae). Acta Zoologica Academiae Scientiarum Hungaricae, 58(3): 259-274.

SZEDERJESI, T. \& CSUZDI, Cs. (2015): A new earthworm species and new records from Albania with remarks on the molecular phylogenetic relationships of the Eisenia species of the Balkan Peninsula (Oligochaeta: Lumbricidae). NorthWestern Journal of Zoology, 11(1): 110-116.

SzEDERJESI, T. \& Misırlığlu, M. (2017): New earthworm records from Turkey (Clitellata: Lumbri- 
cidae, Megascolecidae). Opuscula Zoologica, Budapest, 48(1): 55-60. doi: 10.18348/opzool.2017.1.55

SzEDERJESI, T., PAVlíčEK, T. \& CSUZDI, Cs. (2013a): New earthworm species from Jordan (Oligochaeta, Lumbricidae). Acta Zoologica Academiae Scientiarum Hungaricae, 59(4): 391-400.

SzederJesi, T., PAVlíčEK, T. \& CsuzdI, Cs. (2013b): New data to the earthworm fauna of Israel (Oligochaeta, Lumbricidae). Opuscula Zoologica Budapest, 44(2): 201-209.

SzederJesi, T., PAVlíčEK, T. \& CsuzdI, Cs. (2016): Description of the first endemic earthworm species from Cyprus (Oligochaeta: Lumbricidae). Zoology in the Middle East, 62(2): 158-163. doi: 10.1080/09397140.2016.1182778

SZEDERJESI, T., POP, V.V. \& CSUZDI, Cs. (2014b): New and little known earthworm species from peripheral areas of the Romanian Carpathians (Oligochaeta, Lumbricidae). Acta Zoologica Academiae Scientiarum Hungaricae, 60(2): 85-107.

SzederJesi, T., PAVlíčeK, T., CoşKun, Y. \& Csuzdi, Cs. (2014a): New earthworm records from Turkey, with description of three new species (Oligochaeta: Lumbricidae). Zootaxa, 3764(5): 555-570. doi: 10.11646/zootaxa.3764.5.4

SzederJesi, T., Angyal, D., BALÁzs, G. \& DÁNyi L. (2014c): Remarks on the earthworm genus Helodrilus Hoffmeister, 1845 with new epigean and subterranean records (Oligochaeta, Lumbricidae). Opuscula Zoologica Budapest, 45(2): 181-188.

SzederJesi, T., VAvoulidou, E., Chalkia, C., DÁNYi, L. \& CSUZDI, Cs. (2017a): An annotated checklist of earthworms of Greece (Clitellata: Megadrili). Zootaxa, 4272(1): 057-082. doi: $10.11646 /$ zootaxa.4272.1.3

SzederJesi, T., PoP, V.V., PAVlíčEK, T., MÁrton, O., KRÍzSIK, V. \& CSUZDI, Cs. (2017b): Integrated taxonomy reveals multiple species in the Dendrobaena byblica (Rosa, 1893) complex (Oligochaeta: Lumbricidae). Zoological Journal of the Linnean Society, 181: zlx049 doi: 10.1093/zoolinnean/zlx049

Trakić, T., Valchovski, H. \& Stojanović, M. (2016): Endemic earthworms (Oligochaeta: Lumbricidae) of the Balkan Peninsula: a review. Zootaxa, 4189(2): 251-274. doi: 10.11646/zootaxa.4189.2.3
UDE, H. (1885): Über die Rückenporen der terricolen Oligochaeten, nebst Beiträge zur Histologie des Leibesschlauches und zur Sytematik der Lumbriciden. Zeitschrift für Wissenschaftliche Zoologie, 43: 87-143.

UDE, H. (1922): Regenwürmer aus Mazedonien. Archiv für Naturgeschichte, 88: 155-162.

VALCHOVSKI, H. (2012): Checklist of earthworms (Oligochaeta: Lumbricidae) from Bulgaria - a review. Zootaxa, 3458: 86-102.

VALCHOVSKI, H. (2014): Diversity of earthworms (Oligochaeta: Lumbricidae) in Sofia Plain, Bulgaria. ZooNotes, 59: 1-9.

VAlCHOVSKI, H. \& SZEDERJESI, T. (2016): New and additional records of earthworms (Oligochaeta: Lumbricidae) from Bulgaria: first finding place of endemic species Cernosvitovia munteniana on the Balkan Peninsula. North-Western Journal of Zoology, 12(2): 356-360.

VALCHOVSKI, H. \& MisiRLIOĞLU, M. (2017): Murchieona minuscula (Rosa, 1906) first finding from Bulgaria with earthworm diversity and zoogeography of Y1ldı (Strandja) Mountain in Turkey and Bulgaria. Turkish Journal of Zoology, 41: 731736. doi: 10.3906/zoo-1605-46

WAGA, A. (1857): Sprawozdanie $\mathrm{z}$ podrozy naturalistov odbytej w r. 1854 do Ojcowa. Bibliotheca Warszawie, 2: 161-227.

ZICSI, A. (1963): Ein neuer Regenwurm aus der Gattung Lumbricus (Oligochaeta). Zoologischer Anzeiger, 170: 73-76.

ZICSI, A. (1967): Beiträge zur Kenntnis der Ungarischen Lumbricidenfauna, V. Acta Zoologica Academiae Scientarum Hungaricae, 13: 245-252.

ZICSI, A. (1969): Beitrag zur Revision der Regenwurm-Sammlung Karl Wesselys im OÖ. Landesmuseum zu Linz. Naturkundliches Jahrbericht der Stadt Linz, 15: 69-76.

ZICSI, A. (1970): Bemerkungen zum Problem von Octolasium (Octodrilus) croaticum (Rosa, 1895), nebst Beschreibung von zwei neuen Arten der Untergattung Octodrilus (Oligochaeta: Lumbricidae). Opuscula Zoologica Budapest, 10: 165-174.

ZICSI, A. (1971): Regenwürmer aus dem Tessin sowie Bemerkungen über die meroandrischen Formen der Untergattung Octodrilus (Oligochaeta: Lumbricidae). Acta Zoologica Academiae Scientiarum Hungaricae, 17: 219-231. 
ZICSI, A. (1973): Regenwürmer (Oligochaeta: Lumbricidae) aus der Türkei. Acta Zoologica Academiae Scientiarum Hungaricae, 19: 217-232.

ZICSI, A. (1974): Ein neue Dendrobaena-Art (Oligochaeta: Lumbricidae) aus Griechenland. Acta Zoologica Academiae Scientiarum Hungaricae, 20: 449-451.

ZICSI, A. (1981a): Probleme der Lumbriciden-Systematik sowie die Revision zweier Gattungen (Oligochaeta). Acta Zoologica Academiae Scientiarum Hungaricae, 27: 431-442.

ZICSI, A. (1981b): Weitere Angaben zur Lumbricidenfauna Italiens (Oligochaeta: Lumbricidae). $O$ puscula Zoologica Budapest, 17-18: 157-180.

ZICSI, A. (1985a): Regenwürmer (Oligochaeta: Lumbricidae) aus Israel und den benachbarten Ländern. Revue Suisse de Zoologie, 92: 323-331.

ZICSI, A. (1985b): Über die Gattungen Helodrilus Hoffmeister, 1845 und Proctodrilus gen. n. (Oligochaeta: Lumbricidae). Acta Zoologica Academiae Scientiarum Hungaricae, 31: 275-289.
ZICSI, A. \& CSUZDI, Cs. (1986): Regenwürmer aus Bulgarien (Oligochaeta Lumbricidae). Opuscula Zoologica Budapest, 22: 113-121.

ZICSI, A. \& MiCHALIS, K. (1981): Übersicht der Regenwurm-fauna Griechenlands (Oligochaeta: Lumbricidae). Acta Zoologica Academiae Scientiarum Hungaricae, 27: 239-264.

ZICSI, A. \& MiCHALIS, K. (1993): Zwei neue Dendrobaena-Arten aus Grichenland (Oligochaeta: Lumbricidae). Acta Zoologica Academiae Scientiarum Hungaricae, 39: 301-310.

ZICSI, A., PoP, V.V. (1991): Cernosvitovia munteniana sp. n. ein neuer Regenwurm aus Rumanien (Oligochaeta, Lumbricidae). Mitteilungen aus den Hamburgischen Zoologischen Museum und Institut, 88: 125-127.

ZICSI, A. \& ŠAPKAREV, J. (1982): Eine neue Cernosvitovia-Art aus Jugoslawien (Oligochaeta: Lumbricidae). Acta Zoologica Academiae Scientiarum Hungaricae, 28: 181-182. 